\title{
Geschlecht und gesundheitliche Ungleichheiten - Soziale Netzwerke im Kontext von Gesundheit und Gesundheitsverhalten
}

\author{
Markus Gamper, Julia Seidel, Annett Kupfer, \\ Sylvia Keim-Klärner und Andreas Klärner
}

\section{Überblick}

- Es gibt deutliche Unterschiede hinsichtlich der Morbidität (Krankheitshäufigkeit) und der Mortalität (Sterblichkeit) zwischen Männern*1 und Frauen*.

\footnotetext{
${ }^{1}$ Mit der Verwendung des ,*“ weisen die Autor*innen zum einen auf den Konstruktionscharakter der Kategorie Geschlecht hin und sie möchten zum anderen aufzeigen, dass es sich bei der Geschlechtsidentität und dem Geschlechtsausdruck nicht um abgeschlossene
}

M. Gamper $(\bowtie)$

Köln, Deutschland

E-Mail: m.gamper@uni-koeln.de

J. Seidel · A. Kupfer

Dresden, Deutschland

E-Mail: julia.seidel4@tu-dresden.de

A. Kupfer

E-Mail: annett.kupfer@tu-dresden.de

S. Keim-Klärner · A. Klärner

Braunschweig, Deutschland

E-Mail: sylvia.keim-klaerner@thuenen.de

A. Klärner

E-Mail: andreas.klaerner@thuenen.de 
- Bis zur Pubertät weisen männliche* Jugendliche häufiger gesundheitliche Probleme auf.

- Während der Pubertät leiden Mädchen* an chronischen und psychischen Erkrankungen und männliche* Heranwachsende eher an akuten und lebensbedrohlichen Krankheiten (z. B. HIV).

- Jungen* und Männer* haben ein riskanteres Gesundheitsverhalten.

- Das Forschungsfeld bezieht sich hauptsächlich auf die Binarität der Geschlechter Mann und Frau. Studien zu trans ${ }^{* 2}$ und queren ${ }^{3}$ Personen sind in diesem Bereich selten.

- Netzwerke haben einen geschlechtsspezifischen Effekt auf das Risikoverhalten.

- Frauen* leisten mehr und zeitintensivere soziale Unterstützung, auch im Krankheitsfall.

- Nach der Verwitwung haben Netzwerke sowohl negative wie auch positive Effekte. Diese sind geschlechtsspezifisch.

Kategorien handelt, sondern auch, jenseits der Binarität von weiblich* und männlich* bzw. Frau* und Mann*, eine Vielfalt existiert und es Menschen gibt, die sich nicht eindeutig der dualistischen Geschlechterordnung zuordnen wollen oder können. Mittlerweile gibt es zwar eine wachsende Zahl an Studien speziell zu trans* Personen, das Geschlecht wird aber in der Mehrheit der allgemeinen Studien als dichotome Variable (,männlich“/ „weiblich“) abgefragt (Döring 2013). Wir fokussieren uns in diesem Aufsatz auf Studien mit dichotomen Geschlechtervariablen, lassen aber wenn möglich auch Befunde aus der Forschung zu trans* Personen einfließen. Zum anderen weist das ,"* darauf hin, dass bei Bezeichnungen von Personengruppen, ausdrücklich sowohl weibliche als auch männliche Personen gemeint sind sowie alle Menschen, die sich nicht eindeutig der dualistischen Geschlechterordnung zuordnen wollen oder können.

${ }^{2}$ Die (Selbst-)Bezeichnung trans* stellt einen Oberbegriff für verschiedene, mit „Trans“ beginnende Bezeichnungen dar, zum Beispiel Transgender, Transidentität und Transsexualität usw. Zudem nimmt das , ${ }^{*}$ “ die Rolle eines*einer Platzhalter*in für vielfältige (Selbst-)Bezeichnungen ein (Gerede e. V. 2018).

${ }^{3}$ Queer bezeichnet vor allem Personen, die sich nicht mit traditionellen Geschlechterrollen und -stereotypen identifizieren und eine behauptete Zweigeschlechtlichkeit infrage stellen. Queer steht aber auch für Menschen, die durch ihre Selbstdefinition (trans*, multisexuell, lesbisch etc.) Heteronormativität ablehnen (i-Päd 2019). 


\section{$1 \quad$ Geschlecht als Ungleichheitskategorie}

Geschlecht gehört mittlerweile zu einer der wichtigsten Kategorien im sozialwissenschaftlichen Diskurs. Die Geschlechtskategorie durchzieht und prägt alle gesellschaftlichen Lebensbereiche und nimmt in der Funktion einer horizontalen Ungleichheitsdimension eine bedeutungsvolle Rolle als soziale Ordnungs- und gesellschaftliche Positionierungskategorie ein (Babitsch 2009; Rose 2015). Sowohl für das weibliche* als auch für das männliche* Geschlecht ,existieren geschlechtsspezifische Normalitätsvorstellungen, Verhaltensstandards und Inszenierungsskripte, mit denen sie sich im Laufe ihres Lebens unentwegt arrangieren" müssen (Rose 2015, S. 63). In unserem Alltagswissen wird die Zweigeschlechtlichkeit des Menschen sowie die von Geburt (und größtenteils Schwangerschaft) an festgelegte Geschlechtszugehörigkeit und die dazu implizierte Heterosexualität meist als selbstverständlich und als naturgegeben akzeptiert sowie praktiziert (Wetterer 2004).

Jedoch handelt es sich bei der Geschlechtskategorie um ein sozialstrukturelles und sozial konstruiertes sowie historisch und gesellschaftlich gewachsenes Phänomen, das in sozialen und alltäglichen Interaktionen sowie Handlungen (re)produziert wird (doing gender). Geschlecht ordnet Individuen in zwei unterschiedliche Gruppen, die sowohl auf einer biologisch gebundenen Zuordnung, als auch auf gesellschaftlichen Zuschreibungsprozessen beruhen (Winker und Degele 2010).

Als wichtiger Schritt in der Betrachtung und Analyse des Geschlechts gilt die Trennung in die zwei Komponenten sex (das bei der Geburt aufgrund biologischer Merkmale zugewiesene Geschlecht) und gender (als soziale, kulturelle Dimension und Geschlechtsidentität) und die damit verbundene Möglichkeit „die mit dem biologischen Geschlecht verbundene Vorstellung von naturgegebenen Unterschieden zwischen Frauen und Männern in Frage zu stellen und im Kontext der gesellschaftlichen Herstellungsmodi zu reflektieren“ (Babitsch 2009, S. 284). Die geschlechtsspezifischen Verhaltensweisen unterliegen somit einer sozialen Prägung und sind zudem abhängig von den jeweiligen kulturellen, historischen und gesellschaftlichen Bedingungen. Des Weiteren können die existierenden gesellschaftlichen Geschlechterungleichheiten nicht alleine durch die biologischen Geschlechterdifferenzen begründet werden (Babitsch 2009; Degele 2010).

\subsection{Geschlecht und Gesundheit}

Seit den 1970er Jahren hat das Thema Geschlecht und dessen Einfluss auf die Gesundheit sowohl in der Forschung als auch in der medizinischen Praxis an 
Einfluss gewonnen (Babitsch 2009; Kuhlmann 2016). Durch eine Vielzahl an Studien kann ein mitunter stark ausgeprägter Geschlechterunterschied bezüglich des Gesundheitsgeschehens nachgewiesen werden, d. h., in der Morbidität (Krankheitshäufigkeit) und der Mortalität (Sterberate), in den Entstehungsprozessen von Krankheiten sowie den Krankheitsverläufen und im Gesundheitsverhalten scheinen Männer* und Frauen* sich signifikant zu unterscheiden (z. B. Robert-Koch-Institut 2015). Studien zu Trans*-Personen sind eher selten. Meist umfassen vorliegende Forschungen die ganze Gruppe LGBT*I*Q: „Lesbian, Gay, Bisexual, Transgender, Intersexual and Queer".

\section{Lebenserwartung und Mortalität}

Weltweit lässt sich in den meisten Ländern ein homogenes Muster hinsichtlich der Lebenserwartung ausmachen: die Lebenserwartung der Frauen* ist in der Regel höher als die der Männer* (Kolip und Hurrelmann 2002). ${ }^{4}$ Auch in Deutschland liegt die mittlere Lebenserwartung von Frauen* bei der Geburt derzeit bei 83,1 Jahren, jene von Männern* bei 78,3 Jahren (Robert-Koch-Institut 2015; Statistisches Bundesamt 2019e). Dabei nähern sich die Geschlechterdifferenzen in den letzten Jahren zugunsten des männlichen* Geschlechts an (Kolip und Hurrelmann 2002; Lampert et al. 2017), was auf den Anstieg gesundheitsriskanten Verhaltens unter Frauen* (z. B. steigende Anzahl rauchender Frauen*) zurückgeführt wird. Die kürzere Lebenserwartung des männlichen* Geschlechts besteht jedoch weiter fort. So zeigen weltweit auch männliche* Säuglinge ein höheres Sterberisiko als weibliche* Säuglinge (WHO 2019). Die ungünstige Sterblichkeitsstatistik des männlichen* Geschlechts setzt sich auch im weiteren Lebenslauf fort und wird im Alter zwischen 25 bis 65 Jahren besonders deutlich. In Deutschland starben 2016 fast doppelt so viele Männer* (86.654) im mittleren Lebensalter wie Frauen* (46.815) (Statistisches Bundesamt 2019a). Als Ursache für den Geschlechtsunterschied bzgl. der Übersterblichkeit wird häufig ein gesundheitsriskanteres Verhalten der Männer* angeführt (Hurrelmann und Quenzel 2011; Kolip und Hurrelmann 2002; Sieverding 2005; Robert-KochInstitut 2015). Babitsch (2009) führt ergänzend dazu aus, dass eine Vielzahl an

\footnotetext{
${ }^{4}$ Für die Jahre 2015-2020 konnten die Vereinten Nationen exemplarisch folgende Lebenserwartungen, in Jahren gemessen, ermitteln: Afghanistan (Männer*( $\left.\mathbf{M}^{*}\right)$ : 62,7; Frauen* (F*): 65,6); Brasilien (M*: 72,2; $\left.\mathrm{F}^{*}: 79,4\right)$; Japan (M*: 80,7; $\left.\mathrm{F}^{*}: 87,1\right)$; Canada (M*: 80,7; $\left.F^{*}: 84,4\right)$; Estland $\left(M^{*}: 73,01 ; F^{*}: 81,9\right)$; Kenia $\left(M^{*}: 64,9 ; F^{*}: 69,6\right)$; Neuseeland (M*: 80,4; F*: 83,7) (UNdata 2017).
} 
Studien auf größere sozioökonomisch bedingte Unterschiede in der Lebenserwartung von Frauen* und Männern* hindeuten.

Auch bei den diversen Todesursachen ergeben sich deutliche Unterschiede der Geschlechter. Frauen* sterben häufiger an Herz-Kreislauf-Erkrankungen. Jedoch sterben sie seltener an bösartigen Neubildungen. ${ }^{5}$ Bei Atmungs- oder Verdauungssystemerkrankungen findet man nur geringfügige Geschlechtsdifferenzen (Robert-Koch-Institut 2015). Ein enormer geschlechtsspezifischer Unterschied kann für die Suizidrate bestätigt werden. Im Jahr 2016 beendeten 9838 Menschen ihr Leben, wobei der Anteil der Männer* mit $75 \%$ dreimal so hoch war wie der Anteil der Frauen* mit 25 \% (Statistisches Bundesamt 2019b, c).

\section{Morbidität im Lebenslauf}

Auch bei der Morbidität werden geschlechtsspezifische Differenzen sichtbar. Hurrelmann und Quenzel (2011) konstatierten, dass bereits im Kindesalter, d. h. vom ersten Lebensjahr bis zum Eintreten der Geschlechtsreife im Alter von etwa zwölf Jahren, gesundheitliche Unterschiede zwischen den Geschlechtern auftreten: Jungen* schneiden in den meisten Gesundheitsindikatoren schlechter ab als Mädchen*. Folglich wurden im Jahr 2017 bis zu dem Alter von 15 Jahren mehr Jungen* (4598) als Mädchen* (3659) wegen gesundheitlicher Störungen von ärztlichem Fachpersonal untersucht (Hurrelmann und Quenzel 2011; Robert-Koch-Institut 2015; Statistisches Bundesamt 2019a). Auch in der Krankheitshäufigkeit können geschlechtsspezifische Unterschiede im Kindesalter aufgezeigt werden (Hurrelmann und Quenzel 2011; Kolip und Hurrelmann 2002). Exemplarisch erkrankten laut dem Statistisches Bundesamt (2019a) im Jahr 2017 Jungen* im Alter von 1-15 Jahren häufiger als Mädchen* an Leukämie, an Epilepsie, an chronischen Krankheiten der unteren Atemwege, wie zum Beispiel Asthma bronchiale, und leiden häufiger an Übergewicht sowie Schlafstörungen. Eine größere Anfälligkeit der männlichen* Geschlechter wird sich ebenfalls im Bereich der psychischen Krankheiten konstatiert (Statistisches Bundesamt 2019a; Robert-Koch-Institut 2015; Hurrelmann und Quenzel 2011).

Erst mit dem Einsetzen der Pubertät verändert sich diese Situation innerhalb weniger Jahre. Mädchen* im Alter ab 15 Jahren erkranken signifikant häufiger

\footnotetext{
${ }^{5}$ Auch bei der Art der bösartigen Neubildungen zeigen sich Geschlechtsdifferenzen. Laut dem Statistischen Bundesamt (2019i) kann für das Jahr 2016 für die männlichen* Geschlechter Lungen- und Bronchialkrebs als die häufigste Krebserkrankung mit Todesfolge aufgeführt werden, mit 29.305 von 125.128 Fällen. Bei den Frauen* ist es mit 18.570 von 105.597 Fällen der Brustkrebs.
} 
als Jungen* an psychosomatischen und physiologischen Beschwerden (Kolip und Hurrelmann 2002): Mädchen* im Alter von 15-18 Jahren leiden beispielsweise öfter als Jungen* an Kopfschmerzen, an Bauch- und Beckenschmerzen, an Schwindel und Taumel und an Essstörungen (Statistisches Bundesamt 2019a). Mit Beginn der Pubertät ändert sich zudem die Inanspruchnahme von ärztlicher Konsultation und Behandlung, die nun mehr Mädchen* als Jungen* in Anspruch nehmen (Hurrelmann und Quenzel 2011; Statistisches Bundesamt 2019a).

Die Pubertät kennzeichnet zwar eine Kehrtwende sowohl in der Inanspruchnahme von medizinischen Hilfeleistungen als auch im Gesundheits- und Krankheitsgeschehen, die geschlechtsspezifische Tendenz setzt sich jedoch im Erwachsenenalter (18-65 Jahre) fort (Kolip und Hurrelmann 2002). Diesbezüglich konstatieren das Robert-Koch-Institut (2015), Lampert et. al (2017) sowie Babitsch (2009) einen geschlechtsspezifischen Unterschied in der subjektiven Selbsteinschätzung der Gesundheit. 72,9\% aller Frauen* im mittleren Alter bewerten ihre Gesundheit als gut oder sehr gut, hingegen sind es bei den Männern* 76,6 \% (Robert-Koch-Institut 2015).

Das Vorhandensein einer oder mehrerer chronischer Erkrankungen führt ebenfalls zu einer geschlechtsspezifischen Differenz. Kolip und Hurrelmann (2002), Sieverding (2005) und Regitz-Zagrosek (2018) heben hervor, dass das weibliche* Krankheitsprofil eher durch chronische Erkrankungen sowie durch psychosomatische und psychische Beeinträchtigungen (z. B. Schilddrüsenerkrankungen, Depression, Essstörungen, Migräne, Hypertonie, Gallenblasenerkrankungen, Arthrose, Osteoporose, koronare Herzerkrankungen) geprägt ist; das männliche* hingegen durch akute und lebensbedrohliche Krankheiten (z. B. HIV-Infektion, bösartige Neubildungen der Verdauungsorgane sowie der Lungen und Bronchien, Lungenemphysem, Leberzirrhose, koronare Herzerkrankungen). Eine Metaalanalyse zu Trans*-Personen weist darauf hin, dass insbesondere Trans*männer ${ }^{6}$ stark von HIV/AIDS betroffen sind. Hierbei gibt es auch Zusammenhänge mit anderen Risikofaktoren wie beispielsweise Prostitution oder Diskriminierung (Herbst et al. 2008). Insgesamt zeigt sich bei LGBT*I*Q-Personen ein hohes Risiko für Darmerkrankungen (z. B. Giardien, Amöben), Hepatitis A und B, Humane Papillomviren ${ }^{7}$ und Analkarzinom (Analkrebs) (Dean et al. 2000).

\footnotetext{
${ }^{6}$ Personen, die sich als überwiegend männlich identifizieren, welche aber bei der Geburt dem weiblichen Geschlecht zugeordnet wurden (Gerede e. V. 2018).

${ }^{7}$ Diese Viren können Haut sowie verschiedener Schleimhäute infizieren und ein unkontrolliertes tumorartiges Wachstum hervorrufen.
} 
Eine besondere Bedeutung in Bezug auf geschlechtsspezifische Differenzen hinsichtlich der Morbidität nehmen zudem die koronaren Herzerkrankungen (KHK) ein, der nach Kuhlmann (2016) unter dem Geschlechtsaspekt beste untersuchte Bereich. Laut Kuhlmann (2016) und Regitz-Zagrosek (2017, 2018) existieren geschlechtsspezifische Unterschiede in fast jedem Studium einer KHK. So führt Regitz-Zagrosek (2017) beispielsweise Differenzen bei den Risikofaktoren an. ${ }^{8}$ Weitere Beispiele bringt Kuhlmann (2016) ein und bezieht sich auf deutsche und internationale Studien. Demnach werden „Frauen mit KHK seltener evidenzbasiert behandelt [...], [erhalten] seltener invasive Diagnostik, [werden] seltener und anders über präventive Maßnahmen (z. B. Raucherentwöhnungsprogramme) aufgeklärt" (Kuhlmann 2016, S. 189).

Auch in Bezug auf psychische Erkrankungen können Differenzen zwischen den Geschlechtern verzeichnet werden. Laut den Daten des Robert Koch-Instituts (2015) liegt die Prävalenz für Angststörungen bei Frauen* mit 21,3 \% höher als bei Männern* mit 9,3\%. Ein ähnliches Bild zeigt sich bei den depressiven Störungen und bei chronischem Stress (Hapke et al. 2013). Auch wenn es insgesamt kaum Forschungen zu vollendetem Selbstmord sowie Suizidgefahren unter Trans*-Personen gibt, kann das sehr ausführliche Review von Haas et al. (2010) eine erhöhte Suizidgefahr wie auch eine erhöhte Anzahl an vollendeten Selbstmorden für trans*-Personen nachweisen. Dies gilt wohl besonders für junge Heranwachsende (Mustanski et al. 2010). Trans*-Menschen weisen zudem im Vergleich zur Allgemeinbevölkerung höhere Prävalenzraten u. a. für Depressionen und Angsterkrankungen auf (Nieder et al. 2016).

Bezüglich Alkoholabhängigkeit scheinen Männer* stärker als Frauen* betroffen zu sein. Pabst et al. (2013) konstatieren für das Jahr 2012 eine Alkoholabhängigkeit bei 2,0 \% der Frauen* und 4,8 \% der Männer* in Deutschland. Im Jahr 2017 registrierte die Krankenhausdiagnosestatistik bei Frauen* 85.283 und bei Männern* 228.928 stationäre Behandlungsfälle aufgrund psychischer und Verhaltensstörungen durch Alkohol (Statistisches Bundesamt 2019a).

\footnotetext{
${ }^{8}$ Geschlechtsspezifische Unterschiede im Zusammenhang mit Risikofaktoren existieren bei dem Einfluss von Diabetes mellitus, der bei Frauen* stärker ausgeprägt ist als bei Männern*. Laut Regitz-Zagrosek (2017) erhöht Diabetes mellitus das Auftreten einer KHK bei Frauen* um das 5- bis 7-fache und bei Männern* um das 3- 4-fache. Die klassischen Risikofaktoren wie Hypertonie, Zigarettenrauchen, Hypercholesterinämie haben bei dem weiblichen* Geschlecht hingegen eine ähnliche Auswirkung auf das KHK-Risiko wie bei dem männlichen* Geschlecht.
} 
Im hohen Alter ist kaum ein genereller Geschlechterunterschied zu erkennen. Hinsichtlich der Multimorbidität schneidet das weibliche* Geschlecht signifikant schlechter ab, d. h., Frauen* leiden mit zunehmendem Alter öfter als Männer* unter multiplen chronischen Erkrankungen, z. B. Osteoporose, Osteoarthrose und Herzinsuffizienz. Bei dem männlichen* Geschlecht treten auch im hohen Alter eher lebensbedrohliche Erkrankungen, wie Herzinfarkte und chronisch obstruktive Lungenerkrankungen auf (Iller und Wienberg 2012; Statistisches Bundesamt 2019a).

Auch im Hinblick auf die subjektive Beurteilung des eigenen Gesundheitszustandes können weiterhin Geschlechtsunterschiede belegt werden: Männer* sind im Allgemeinen zufriedener; mit zunehmenden Alter sinkt die Gesundheitszufriedenheit jedoch schneller ab (Iller und Wienberg 2012).

Für Trans*- Personen fassen Graham et al. (2011) einzelne Aspekte in den verschiedenen Lebensphasen zusammen, können aber nach eigenen Angaben kaum Ergebnisse liefern.

\subsection{Geschlecht und Gesundheitsverhalten}

Laut Kuhlmann (2016) beeinflussen kulturelle und soziale Faktoren die Inanspruchnahme von gesundheitlichen Leistungen und führen teilweise $\mathrm{zu}$ signifikanten Geschlechterdifferenzen in fast allen Bereichen des gesundheitlichen Versorgungssystems. Beispielsweise nehmen mehr Frauen* an Gesundheitskursen der Volkshochschule und der Krankenkassen teil (Kuhlmann 2016; Robert-Koch-Institut 2015), die allerdings vermutlich auch primär auf die Bedürfnisse der Frauen* abzielen und somit Männer* benachteiligen (Kuhlmann 2016).

Auch bei der Inanspruchnahme von ambulanter ärztlicher Versorgung kann laut Prütz und Rommel (2017) teilweise ein erheblicher Unterschied, aber auch eine deutliche Angleichung der Geschlechter mit zunehmendem Lebensalter verzeichnet werden. Ein Geschlechtsunterschied zeigt sich insbesondere im jungen Erwachsenenalter: So nahmen von den 18- bis 29-jährigen Frauen* 90,4 \% und lediglich 78,4 \% der Männer* in den letzten zwölf Monaten eine Leistung in Anspruch. Bei den 65-Jährigen und älteren Personen sind jedoch kaum mehr Unterschiede feststellbar. Neuere Studien sowie differenzierte Analysen widerlegen zudem laut Kuhlmann (2016) die Annahme, dass sich Männer* weniger um ihre Gesundheit kümmern.

Die Inanspruchnahme von psychiatrischen und psychotherapeutischen Leistungen nimmt im Kontext der Geschlechterdifferenzen eine besondere Stellung 
ein. Laut Barry und Yuill (2012) und Rommel et al. (2017) zeigen Männer* hier besonders große Zurückhaltung bei der Konsultation bzw. Meldung einer psychischen Krankheit, was die Autor*innen auf das vorherrschenden gesellschaftlichen Geschlechterstereotypen zurückführen (Barry und Yuill 2012).

Rauchverhalten ist einer der bedeutenden Risikofaktoren für Gesundheit und die führende Ursache vorzeitiger Sterblichkeit. Im Geschlechtervergleich zeigt sich laut Zeiher et al. (2017) und hier den Daten der GEDA-Studie 2014/2015-EHIS ein leicht stärkeres (gelegentliches) Rauchverhalten bei Männern* $(27,0 \%)$ als bei Frauen* (20,8\%). Laut der Robert-Koch-Stiftung (2015) hat sich die Rauchquote der Geschlechter durch die Zunahme weiblicher* Raucherinnen in den letzten 20 Jahren jedoch angenähert. Als Erklärungsursache für den Anstieg der weiblichen* Rauchquote werden veränderte Geschlechterrollen und-stereotypen angenommen (Sieverding 2005; Kolip und Hurrelmann 2016; Bartley 2017, s. u.).

Alkoholkonsum weist als Risikofaktor erhebliche geschlechtsspezifische Ungleichheiten in allen Lebensaltern auf: So berichten Orth und Töppich (2015), dass im Jahre 2014 8,5\% der Mädchen* im Alter von 12 bis 17 Jahren und 14,9\% der Jungen* im selben Alter in den letzten zwölf Monaten mindestens einmal wöchentlich riskante Mengen an Alkohol konsumiert haben. Auch im Erwachsenenalter zeigt sich diese Geschlechtsdifferenz (Lange et al. 2017). Dabei ist ein signifikanter Einfluss von sozialen Unterschieden und dem Alkoholkonsum auf die Geschlechter nachweisbar (Lange et al. 2017). So weisen Frauen* der oberen Bildungsgruppe in allen Altersgruppen eine höhere Prävalenz für riskanten Alkoholkonsum auf als Frauen* aus unteren Bildungsgruppen; bei dem männlichen Geschlecht* trifft ähnliches zu.

Kaum eine Forschung hat den Substanzkonsum bei trans*-Jugendlichen und -Heranwachsenden untersucht. Meist beziehen sich vorhandene Studien - wie bereits erwähnt - auf die Gruppe der LGBT*I*Q-Personen. Eine Studie über junge LBT*I*Q-Frauen* in San Francisco zeigt allerdings, dass Substanzkonsum bei trans*weiblichen ${ }^{9}$ Jugendlichen sehr verbreitet ist und dies signifikant mit psychosozialen Risikofaktoren in Verbindung steht (Rowe et al. 2015). Eine Langzeitstudie in den USA konnte daran anschließend nachweisen, dass der Alkoholkonsum im Laufe der Zeit linear zunahm. Bei männlichen* LGBT*I*Q-Jugendlichen* konnte hierbei eine tendenziell schnellere Zunahme verzeichnet werden als bei den weiblichen* Jugendlichen (Newcomb et al. 2012).

\footnotetext{
${ }^{9}$ (Selbst-)Bezeichnung von Personen, die sich als überwiegend weiblich identifizieren, aber bei der Geburt dem männlichen Geschlecht zugeordnet wurden (Gerede e. V. 2018).
} 
Der Missbrauch von verschreibungspflichtigen Opioiden und Beruhigungsmitteln zeigt sich bei LGBT*I*Q-Jugendliche schon in jungen Jahren (Kecojevic et al. 2012). Daran anknüpfend finden sich in einer weiteren US-Studie Belege, dass der Missbrauch von verschreibungspflichtigen Medikamenten relativ häufig bei LGBT*I*Q-Erwachsenen vorkommt und stark mit emotionaler Belastung in Verbindung steht (Benotsch et al. 2013).

\subsection{Ausgewählte Erklärungsansätze im Kontext geschlechtsspezifischer Gesundheitsunterschiede}

So klar wie die geschlechtsspezifischen Gesundheitsunterschiede aufgezeigt werden konnten, so schwierig gestaltet sich die Ursachenfindung. Viele Fragen konnten in diesem Zusammenhang noch nicht geklärt werden. Von den verschiedenen, aktuell diskutierten Erklärungsansätzen werden im Folgenden drei vorgestellt.

\section{Geschlechtsspezifische Rollenvorstellungen und -stereotype}

Der Einfluss gesellschaftlicher Konstruktionen von Geschlecht auf die Gesundheit werden in wissenschaftlichen Diskursen bereits seit längerem ausführlich diskutiert (Kolip und Hurrelmann 2016; Kuhlmann 2016; Sieverding 2005; Babitsch 2009; Barry und Yuill 2012; Bartley 2017; Regitz-Zagrosek 2017, 2018 u. a.). Es besteht laut Sieverding (2005) weitestgehend ein Konsens darüber, ,dass die Geschlechterunterschiede in physischer Gesundheit und Krankheit am stärksten durch Geschlechtsunterschiede in gesundheitsrelevanten Verhalten, insbesondere im höheren Risikoverhalten von Männern, begründet sind" (Sieverding 2005, S. 57). Das gesundheitsrelevante Verhalten wird wiederum durch eine große Anzahl an soziokulturellen Faktoren beeinflusst. In diesem Kontext werden gesellschaftlichen Geschlechterrollen und -stereotypen eine Schlüsselfunktion beigemessen (Sieverding 2005). Beispielsweise wird dem weiblichen* Geschlecht im Zusammenhang mit Gesundheit immer noch eine eher sorgende und gesundheitsbewusstere Rolle zugesprochen. Dahingegen basiert die Konstruktion männlichen* Geschlechts weiterhin darauf, gesundheitliche Probleme selbstständig und ohne fremde Hilfe lösen bzw. ertragen zu können oder zu müssen, um somit die Kontrolle der eigenen Leistungsfähigkeit wahren zu können (Kolip und Hurrelmann 2002).

\section{Diskriminierung}

Gesellschaftliche Geschlechterstereotype und -rollen wirken sich auch auf die Beurteilung anderer Personen aus. Es wird vermutet, dass Patient*innen von 
ärztlichem Fachpersonal je nach Geschlechtszugehörigkeit abweichend wahrgenommen und auch behandelt werden. Studien weisen darauf hin, dass ärztliches Fachpersonal männliche* Beschwerden ernster nehmen. Dagegen werden bei dem weiblichen* Geschlecht anscheinend häufiger psychisch bedingte Leiden vermutet und die Behandlung dementsprechend ausgerichtet (Kolip und Hurrelmann 2002; Sieverding 2005; Kuhlmann 2016). Eine qualitative Studie zeigt, dass Klinikpersonal auf die gesundheitlichen Bedürfnisse von Trans*-Patientinnen* oft mit Unsicherheit reagiert, welche sich dann in Stigmatisierung ausdrücken kann. Dies führt bei Trans*-Personen wiederum zur Ansicht, dass sie mit ihren Bedürfnissen nicht verstanden werden (Poteat et al. 2013). Ein Review von 17 Artikeln über die Einstellung von Pflegekräften zu LGBT*I*Q-Patienten bestätigte das Ergebnis der Benachteiligung (Dorsen 2012, zur Diskriminierung siehe auch Grant et al. 2011).

\section{Armut und soziale Ungleichheit}

Armut und soziale Ungleichheit haben zentrale Auswirkungen auf die Gesundheit und Lebenserwartung der Geschlechter und führen zugleich zu geschlechtsspezifischen Unterschieden. Immer noch bekommen Frauen* im Durchschnitt $21 \%$ weniger Gehalt als Männer*, arbeiten mit $49 \%$ fast doppelt so häufig in Teilzeitbeschäftigung wie Männer* mit $11 \%$ und sind weiterhin öfter von Armut betroffen als Männer* (WSI 2017). Diese geschlechtsspezifischen Ungleichheiten haben einen deutlichen Einfluss auf das Gesundheitsgeschehen.

Kuhlmann (2016) bezieht sich in diesem Zusammenhang beispielsweise auf das deutsche Gesundheitssystem, welches sich durch hohe Qualitätsstandards und eine hohe Versorgungsdichte sowie niedrige Zugangsbarrieren auszeichnet. Dennoch existieren systembedingte Barrieren, die zu Unterschieden in den Zugangschancen von Männern* und Frauen* führen. Als Beispiel nennt Kuhlmann (2016) die steigenden finanziellen Belastungen der Nutzer*innen im Zusammenhang mit dem zu erbringenden Eigenanteil bei den Krankenkassenbeiträgen. Der Eigenanteil beträgt aktuell in Deutschland 14,3\%, 2010 lag er noch bei $13 \%$ (WHO 2010; Bundesversicherungsamt 2017). Es ist also eine leichte Steigung zu verzeichnen. „Hier liegt es auf der Hand, dass Frauen als Gruppe aufgrund der Einkommensdifferenzen häufiger und stärker als Männer von einem steigenden Eigenanteil an den gesundheitsbezogenen Kosten betroffen sind" (Kuhlmann 2016, S. 188). Dies gilt noch stärker für die Gruppe der älteren Frauen*, da Frauen* mit durchschnittlich 643 EUR im Monat eine niedrigere Rente beziehen als Männer* (1154 EUR) und somit öfter von Altersarmut betroffen sind (ebd., WSI-Gender Daten Portal 2018). Eine US-amerikanische Studie ergab, dass die Arbeitslosenquote bei Trans*-Personen, im Vergleich zur Allgemeinbevölkerung, 
doppelt so hoch ist. Damit sinkt für sie die Wahrscheinlichkeit, zum einen krankenversichert und zum anderen von einem Unternehmen allgemein versichert zu sein (Grant et al. 2011).

\section{Gender und soziale Netzwerke - Ein Überblick}

Historisch zurückgeblickt ging man in den 1970er Jahren davon aus, dass Frauen* und Männern* unterschiedliche Einstellungen zu sozialen Kontakten besitzen (z. B. Miller 1976), ohne dass bis dahin Netzwerkstudien mit großen Stichproben vorlagen ${ }^{10}$, die diese These unterstützten. Erst in den 1980er Jahren kam es vermehrt zu quantitativen wie qualitativen Forschungsarbeiten mit einer expliziten Genderausrichtung, und Geschlecht gewann auch in der Netzwerkforschung immer mehr an Bedeutung. So ist Geschlecht mittlerweile eine Ungleichheitsvariable, die im Vergleich zu den meisten anderen hier im Band dargebotenen Merkmalen sehr gut untersucht ist. Aufgrund der großen Anzahl an Studien und auch weil Geschlecht innerhalb der quantitativen Netzwerkforschung häufig als eine Kontrollvariable herangezogen wird, kann die vorliegende Aufzählung nicht repräsentativ sein, sondern lediglich einen kurzen Ausschnitt der Forschung wiedergeben und auf empirische Herangehensweisen und Lücken hinweisen. Dabei ist einschränkend anzumerken, dass die Suche nach Unterschieden bis heute primär auf einem binären Differenzschema von Mann*/Junge* vs. Frau*/Mädchen* basiert und dadurch Geschlechtsidentitäten wie z. B. transgender oder queer bisher kaum berücksichtigt werden.

\subsection{Soziale Netzwerke und Lebensalter}

Geschlecht, so die Freundschafts- und Schulforschung, ist für Freundschaftsbildungen eine wichtige Variable. Schon für Vorschulkinder können Martin et al. (2013) zeigen, dass die Wahl der Spielpartner*innen überproportional auf gleichgeschlechtliche Kinder fällt. Auch bezüglich der Netzwerke von

\footnotetext{
${ }^{10}$ Einer der ersten Netzwerkforschungen zu Geschlecht ist die qualitative ethnographische Studie von Bott (1957). Sie untersuchte inwieweit häusliche Arbeitsteilung in Beziehungen von Männern* und Frauen* und deren soziale Netzwerke zusammenhängen. Sie stellte fest, dass nicht etwa Schichtzugehörigkeit oder das Wohngebiet, sondern vielmehr die Unterschiede in den persönlichen Netzwerken einen Effekt auf die traditionelle oder gemeinsame Arbeitsteilung innerhalb der 20 untersuchten Paarbeziehungen hatten.
} 
jungen Heranwachsenden (McPherson et al. 2001) und in den ersten Jahren der Sekundarschule (Lubbers und Snijders 2007) zeigt sich noch eine sehr starke Trennung zwischen den Geschlechtern (hohe Gender-Homophilie), welche in den Jahren zwar abnimmt, aber dennoch erhalten bleibt. Mit dem Älterwerden lösen sich diese homogenen Netzwerke langsam auf und es bilden sich geschlechtsheterogenere Gruppen heraus (Feiring 1999). Studien von Lubbers und Snijders (2007) zeigen ferner einen geringen Anteil an Liebesbeziehungen oder Sexualbeziehungen in der Sekundarstufe, während diese in der High-School (Bearman et al. 2004) stärker ausgeprägt sind. In beiden Studien sind diese Sexual- oder auch Beziehungsnetzwerke vornehmlich heterosexuell geprägt und erhöhen damit den Anteil gegengeschlechtlicher Alteri im Netzwerk.

Für ältere Menschen, und hier beispielhaft in den Familiennetzwerken älterer Mexikaner*innen, zeigen sich in der Studie von Fuller-Iglesias und Antonucci (2016) keine Geschlechterunterschiede (Nähe, Anteile im Netzwerk). Gegensätzlich dazu finden Schwartz und Litwin (2018) anhand des europaweiten Längsschnittsurvey „Health, Ageing, and Retirement in Europe“ $(\mathrm{n}=13,938)$ für über 65-Jährige vor allem für Frauen* eine Zunahme an Netzwerkbeziehungen über die Zeit, die parallel dazu weniger in Familiennetzwerken involviert sind.

\subsection{Lebenszyklus und die Zusammensetzung der sozialen Netzwerke}

Hinsichtlich des Lebenszyklus zeichnen unterschiedliche Forschungsergebnisse folgendes Bild. In der Studie von Fischer und Oliker aus dem Jahr 1983 finden sich einige wenige Unterschiede zwischen den Geschlechtern nach der Jugendphase. So sind Frauen* mehr mit Verwandten in Kontakt, während Männer* mehr Mitarbeiter*innen und Kolleg*innen als Netzwerkpartner*innen benennen. Ein größerer Zusammenhang wird hinsichtlich der Lebenszyklusphasen deutlich. Bei einer frühen Heirat und Elternschaft schrumpfen die Freundschaftsnetzwerke bei Frauen* im Vergleich zu den Männern* stärker. Nach der Geburt verkleinern sich diese im Vergleich zu den Frauen* vor allem bei den Männern*. „Further evidence suggests that this interaction effect can be explained by both structural and dispositional factors, the former working to reduce women's friendships relative to men's in the earlier period and the latter expanding their friendships later on " (ebd., S. 132). Hierbei stellten Munch et al. (1997) fest, dass gesellschaftliche Normen hinsichtlich der Kindererziehung in westlichen Ländern Auswirkungen auf die Netzwerkstrukturen haben. Während die Geburt eines Kindes keinen statistisch signifikanten Einfluss auf die Größe des Netzwerks der Männer* hatte, 
war aber ein signifikanter negativer Einfluss auf die Größe des Netzwerks der Frauen* festzustellen.

\subsection{Geschlechterunterschiede in den Netzwerkstrukturen mit Blick auf die Allgemeinbevölkerung}

Neben Studien zum Thema Lebensphasen existieren seit den 1980er Jahren unterschiedliche Studien, die der Frage nach Geschlechterunterschieden hinsichtlich der sozialen Netzwerke in der Allgemeinbevölkerung nachgehen. $\mathrm{Zu}$ nennen ist hier die vielzitierte Studie von Fischer (1982) „To Dwell Among Friends - Personal Networks in Town and City“, die einen Effekt von Geschlecht auf die Netzwerke herausstellt. „Women tend to be involved in networks with more relatives and to have more intimate ties than did otherwise similar man. Young women, particular mothers, were more constricted in various ways, such as in the number of the "just friend" they had [...]" (Fischer 1982, S. 253).

Hingegen, und im Widerspruch zu Fischer (1982), fanden Gillespie et al. (2015) keine wesentlichen geschlechtsspezifischen Unterschiede in der Anzahl der Freund*innen, der Anzahl der Alteri, mit denen man Geburtstage feiert, intime Angelegenheiten (z. B. Sexualleben) oder Problemen spät in der Nacht diskutiert. Die Anzahl der Freundschaften variierte jedoch erheblich nach Familienstand, Alter und Elternstatus (s. o.). Auffällig ist, dass jede*r der Befragten mindestens eine*n enge*n Freund*in nennen kann.

Weitere Studien mit der gleichen Schwerpunktsetzung griffen auf die Daten des General Social Survey (GSS) aus den USA zurück, um herauszufinden wie die Netzwerke in der US-amerikanischen Bevölkerung beschrieben werden können. Die Untersuchung von Marsden (1987) geht der Frage von Unterschieden hinsichtlich der Variablen Alter, Bildung, „race“, Geschlecht und Größe des Wohnortes etc. nach. Im Ergebnis scheinen die Netzwerke von jungen, gut ausgebildeten und großstädtischen Bewohner*innen am größten. Geschlechtsunterschiede findet man in erster Linie in der Zusammensetzung des Netzwerks aus Verwandten und Nicht-Verwanden; so ist der Anteil der Familienangehörigen bei Frauen* größer. Ähnliche Ergebnisse zeigen sich in einer etwas älteren Untersuchung von Moore (1990). Selbst nach Kontrolle der Variablen in Bezug auf Beschäftigung, sozialen Strukturpositionen, Familie und Alter besaßen Frauen* einen größeren Anteil an Verwandtschaftsbeziehungen und einen kleineren Anteil an Bekanntschaftsbeziehungen im Netzwerk sowie eine größere Vielfalt an Verwandtenbeziehungen als Männer*. Diese Unterschiede werden auf 
unterschiedliche strukturelle Beziehungskontexte bzw. -orte zurückgeführt, die bestimmte Möglichkeiten für und Beschränkungen auf die Formierung von engen sozialen Beziehungen ausüben. Die Geschlechterunterschiede in der Netzwerkzusammensetzung und -struktur verschwinden nämlich, wenn Beschäftigungsund Familienstatus sowie das Alter statistisch kontrolliert werden. Gleichwohl bleibt als empirischer Befund, dass die Netzwerke von Frauen* eine höhere Anzahl, einen höheren Anteil und eine größere Diversität von Verwandtschaftsbeziehungen enthalten als die Netzwerke von Männern*.

Eine Studie in Singapur zeigt zudem, dass Männer* und Frauen* eher auf berufliche Kontakte treffen, die von der jeweils eigenen Geschlechtszugehörigkeit (bipolar: Mann* oder Frau*) dominiert werden. Beispielsweise treffen Frauen* eher auf Krankenpfleger*innen, da sie in der Pflege überrepräsentiert sind, wobei unterschiedliche Lebensphasen darauf Einfluss haben. Ab der Geburt eines Kindes kommen Frauen* dann auch in Kontakt mit Berufsgruppen, in denen sie unterrepräsentiert sind wie z. B. Lehrer*innen, was sich wiederum auf die Zusammensetzung des Netzwerkes auswirkt (Chua et al. 2016).

Der Geschlechter-Aspekt scheint in den letzten Jahren in seiner Wirkung auf die Unterschiede der Netzwerkbildung an Kraft verloren zu haben. Während Frauen* noch etwas größere Netzwerke als Männer* besitzen und mehr Gespräche über wichtige Angelegenheiten mit Verwandten führen, besitzen sie jetzt auch immer mehr Beziehungen außerhalb der Familie. Frauen* haben somit nicht mehr ein deutlich verwandtschaftsorientiertes Diskussionsnetzwerk als Männer* und sind nicht mehr so häufig sozial isoliert (McPherson et al. 2006). Dies wird auch durch Fuller-Iglesias und Antonucci (2016) für 18- bis 99-jährige Mexikaner*innen bestätigt.

Ob wirklich Unterschiede hinsichtlich der Netzwerke existieren, ist mit Blick auf die widersprüchlichen Forschungsergebnisse fraglich. So weisen einige Kritiker*innen bspw. auf den starken Interviewer*in-Effekt bei der Erhebung des GSS hin (Fischer 2009), andere wiederum stellen die verwendeten Namensgeneratoren infrage und merken bspw. an, dass Frauen* vielleicht mehr wichtige Dinge zu besprechen haben als Männer* und daher ggf. auch ein größeres Netzwerk besitzen (Bearman und Parigi 2004).

\subsection{Netzwerkressourcen und Geschlechterunterschiede}

Neben der Strukturbeschreibung gehen viele Studien der Frage nach, welche Ressourcen die Netzwerke zur Verfügung stellen können. Dies geschieht auf unterschiedlichen Ebenen: 
1. Auf einer allgemeinen gesellschaftlichen Ebene. Hier wird versucht zu eruieren, inwieweit sich die Ressourcenverteilung in der allgemeinen Bevölkerung zwischen den Geschlechtern unterscheidet.

2. Auf der Ebene der Organisationen wird untersucht, inwieweit die Einbindung in soziale Netzwerke den - meist beruflichen - Erfolg beeinflusst.

\section{Soziale Unterstützung und Ressourcenverteilung}

Gillespie et al. (2015) zeigen, dass Männer* wie Frauen* gleichermaßen auf emotionale Unterstützung zurückgreifen können. Ähnliches zeigt sich auch bei Moore (1990). Bearman und Parigi (2004) weisen allerdings darauf hin, dass vor dem Hintergrund ,wichtige Dinge zu besprechen“ Frauen* mehr Personen angeben als Männer*. Die Studie zur sozialen Unterstützung von Turner und Marion (1994) stützt sowohl einen Lebenszyklus- als auch einen Gendereffekt: Frauen* geben an, mehr soziale Unterstützung von Mitarbeiter*innen, Verwandten und Freund*innen zu erhalten als Männer*. Widersprüchlich dazu zeigen sich bei Vyncke et al. (2014) bezogen auf das verfügbare Sozialkapital von Frauen* und Männern*. Männer* können signifikant mehr Ressourcen im Netzwerk aktivieren, berichten von mehr potenziellen Unterstützungsbeziehungen und mehr Netzwerkpartner*innen, die gesunde Lebensweisen fördern. Hobfoll und Vaux (1993) schlussfolgern dagegen anhand verschiedener Studien, dass Frauen* stärker in soziale Unterstützungsinteraktionen eingebunden sind, sie geschickter in Supportprozessen agieren und deswegen auch häufig mehr und intimere Beziehungen sowie größere Unterstützungsnetzwerke besitzen. Frauen* verbringen mehr Zeit in sozialen Interaktionen, teilen eher Gefühle und persönliche Sorgen und berichten häufiger von erhaltener sozialer Unterstützung.

Walen und Lachman (2000) stellen in ihrer Untersuchung an 2348 in Zweierbeziehungen eingebundenen Erwachsenen (25-75 Jahren) fest, dass Frauen* von mehr Unterstützung durch Familie und Freund*innen berichten, wohingegen Männer* häufiger Unterstützung von ihrer Partnerin* erhalten. Daneben hält Diewald (1991) anhand der Auswertung fünf repräsentativer Bevölkerungsumfragen fest, dass Frauen* in den meisten Lebensformen mehr Ansprechpartner*innen zur Verfügung haben als Männer*. Dies traf vor allem für ledige, alleinerziehende, geschiedene und verwitwete Frauen* zu. Frauen* suchen, so auch Barker et al. (1990), eher als Männer* Unterstützung von nahen und weiter entfernten Verwandten sowie Freund*innen und Nachbar*innen.

Wen dabei Frauen* und Männer* bei der Hilfeinanspruchnahme bevorzugen, scheint widersprüchlich. Belegen Antonucci et al. (1998) und Lenz (2003), dass jeweils das eigene Geschlecht bei der Supportsuche favorisiert wird, zeigen andere Wissenschaftler*innen auch gegenteilige Erkenntnisse. Zwar berücksichtigen 
Frauen* tendenziell häufiger innerfamiliäre Helferinnen*, wie die Schwester*, oder außerfamiliäre weibliche* Helferinnen, wie die Nachbarin* (Nestmann und Schmerl 1992), - allerdings sind Frauen* generell die zentralen „Geberinnen*“. So werden in der Studie von Veiel und Herrle (1991) sowohl von Studierenden als auch depressiven Patient*innen und Eltern krebskranker Kinder durchschnittlich häufiger Frauen* als Männer* als Unterstützer*innen genannt.

Am eindeutigsten zeigt sich die geschlechtsspezifische Arbeitsteilung zudem hinsichtlich der Hilfe im Krankheitsfall. Sowohl von männlichen* als auch von weiblichen* Befragten wurden Frauen* um ein Vielfaches häufiger als Männer* als Quellen sozialer Unterstützung genannt. Ebenso bedeutsame Unterstützerinnen* sind sie bei Niedergeschlagenheit, Ratschlägen bezüglich wichtiger Lebensveränderungen und Problemen mit dem Partner*/der Partnerin* (Diewald 1991). Auch bei Nestmann und Schmerl (1992) werden Frauen* häufiger als Helferinnen* genannt. Sowohl Männer* als auch Frauen* erhalten den Autor*innen zufolge mehr Hilfe von weiblichen* Helferinnen als von männlichen* Helfern (Mutter* häufiger als Vater*, Tochter* vor Sohn*, Schwester* vor Bruder* genannt). Frauen*, und vor allem Mütter*, gelten deshalb als die zentralen Unterstützungsinstanzen für ihre Familie (ebd.). Unter anderem bei Barker et al. (1990) nahmen Männer* in belastenden Situationen signifikant häufiger ihre Partnerin* als Unterstützerin* in Anspruch. Das Angewiesen-Sein des Mannes* auf die Partnerin* ist zudem bei älteren, über 60-Jährigen besonders ausgeprägt (Diewald 1991).

Frauen* fungieren dabei aber nicht nur für ihre Männer* häufiger als Unterstützer*in, sondern bieten der Studienübersicht Schmids (2014) zufolge häufiger und auch zeitintensiveren Support als Väter* für ihre erwachsenen Kinder. Stehen sie vor allem im Haushalt und bei der Kinderbetreuung hilfreich zur Seite, unterstützen Väter* ihre erwachsenen Kinder eher mit Einkäufen, Reparaturen oder Gartenarbeiten. Mit Blick auf die intergenerationellen Beziehungen sind auch aufseiten der Unterstützungsleistungen der Kinder Geschlechterunterschiede festzuhalten. So pflegen Töchter* häufigeren Kontakt mit ihren Eltern als Söhne*, übernehmen Töchter* in vielen Ländern häufiger körperlich anspruchsvolle und zeitintensive Pflegeleistungen und unterstützen insgesamt mehr. Söhne* helfen ihren Eltern vor allem bei administrativen Aufgaben, Reparaturen oder finanziellen Fragen. Allerdings, so Schmid (2014), finden diese Unterschiede in der Generationenforschung bislang wenig Beachtung, weshalb die „Ursachen geschlechtsspezifischer Unterstützungsmuster noch immer unzureichend erforscht [sind]" (ebd., S. 17)

Netzwerk- wie Support-Studien zu den Lebenswelten von Trans*-Personen sind kaum zu finden. Beispielhaft findet sich jedoch bei Pflum et al. (2015) 
ein bedeutsamer Zusammenhang zwischen sozialer Unterstützung und psychischer Gesundheit für Trans*-Personen: Sowohl für die Teilnehmer*innen des trans*-männlichen Spektrums (TMS) als auch des trans*-femininen Spektrums (TFS) ist die allgemeine soziale Unterstützung signifikant negativ mit Symptomen von Angst und Depression verbunden - d. h., mit zunehmender sozialer Unterstützung nahmen Gefühle der Angst und depressiven Verstimmungen ab. Der negative Zusammenhang zwischen trans*gemeinschaftlicher Verbundenheit und Symptomen der psychischen Gesundheit war jedoch nur für TFS-Teilnehmer*innen signifikant.

\section{Soziale Beziehungen von Männern* und Frauen* in Organisationen}

Neben dem allgemeinen Nutzen der Netzwerke vor dem Hintergrund der Sozialkapital- und sozialen Unterstützungstheorien, existieren Forschungsarbeiten über den Unterschied der „Verwertung“ von sozialen Beziehungen zwischen Männern* und Frauen* in Organisationen wie z. B. Wirtschaftsbetrieben oder Universitäten. Hierbei wird davon ausgegangen, dass der berufliche „Erfolg“ nicht nur von Kompetenzen, sondern auch von Netzwerken abhängig ist. Besonders Frauen* scheinen in diesem Fall - u. a. durch Prozesse der Stereotypisierung (Oehlendieck 2003) - benachteiligt zu sein (Lyness und Thompson 2000). Der größte Teil dieser Forschungen verdeutlicht, dass Männer* größere arbeitsbezogene Netzwerke haben, mit größeren Clustern verbunden sind und mehr Vorteile aus diesen Beziehungen ziehen, da Männer* höhere Positionen in hierarchischen Strukturen einnehmen (McGuire 2000).

Im Gegensatz dazu scheinen Frauen* in kleinere und weniger vielfältige Netzwerke eingebettet zu sein, die kaum Ressourcen zur Verfügung stellen. Diese weisen eine weibliche* Homophilie auf und sind vor allem durch Personen aus niedrigeren hierarchischen Positionen besetzt. Da die Subcluster ebenfalls tendenziell homogener sind, gibt es Überlappungen der Ressourcen, was zu einer Sozialkapitalbenachteiligung und Reproduktion der Positionen innerhalb des Netzwerkes führen kann (Lin 2000). Dabei schlussfolgern Scheidegger und Osterloh (2003) kontrastierend dazu, dass überwiegend Männer* (als Personen mit starker Legitimation) aus strukturellen Löchern Karrierevorteile ziehen würden und Frauen* für einen Aufstieg innerhalb der Organisation eher kohäsive, redundante Netzwerke benötigten. Zugleich sind Frauen*, solange nur wenige weibliche* Personen in zentralen statushöheren Positionen vertreten sind, aus ressourcenökonomischen Gründen auf Netzwerkkontakte mit höherrangigen Männern* angewiesen und müssen daher ihre Netzwerkkontakte differenzieren - mit entsprechenden Kosten. In einem Studienüberblick zeigen sie zudem die starke Homophilie der jeweiligen Netzwerke (Manager*innen, Mitarbeiter*innen 
aus Medienunternehmen), wobei v. a. für Männer* galt, dass ihre Netzwerke vorrangig aus ,same sex ties“ bestanden. Es wird deshalb davon ausgegangen, dass Frauen* eher auf ihre individuellen Kompetenzen statt auf das Sozialkapital fokussieren (Poole und Bornholt 1998), während Männer* stärker auf Netzwerke setzen und die Ressourcen besser nutzen (Van Emmerik 2006).

\section{Geschlecht, soziale Netzwerke und gesundheitliche Ungleichheiten}

\subsection{Wirkung von Sozialkapital und sozialer Unterstützung auf gesundheitliche Ungleichheiten}

Die Bedeutung von Geschlecht in der Forschung zu gesundheitlichen Ungleichheiten ist in der letzten Zeit wiederholt hervorgehoben worden und wird in der Regel zentral mit dem Konzept des sozialen Kapitals bzw. der sozialen Unterstützung als einer zentralen Funktion und wichtigem Wirkmechanismus sozialer Netzwerke verknüpft (vgl. den Beitrag: Soziale Beziehungen, soziales Kapital und soziale Netzwerke und den Beitrag: Wirkmechanismen in sozialen Netzwerken). Der Begriff des Netzwerkes wird dann, wenn er überhaupt vorkommt, als Metapher für unterstützende oder für ,Rückhalt“ bietende Beziehungen verwendet.

Es gibt zahlreiche Hinweise darauf, dass dieses soziale Kapital und die Verfügbarkeit sozialer Unterstützung zwischen Männern* und Frauen* ungleich verteilt und dass auch die Wirkung geschlechtsspezifisch zu unterscheiden ist. Dies wurde im vorangegangenen Kapitel bereits zum Teil erörtert (siehe oben). Auf Basis mehrerer Studien geht Underwood (2005) davon aus, dass Frauen* bei Krankheiten (Bypass-Operation, Myokardinfarkt) generell mehr Unterstützung erhalten als Männer*. Sie empfangen oft über einen längeren Zeitraum mehr emotionale, jedoch nicht unbedingt materielle Unterstützung (ebd., siehe auch Hobfoll und Vaux 1993). Die Wirkung wird dagegen unterschiedlich bewertet. In einer finnischen Studie wurde etwa ein positiver Zusammenhang zwischen Vertrauen (trust) bei Frauen* sowie Freizeitaktivitäten mit anderen bei Männern* und einer niedrigeren Mortalität festgestellt. Basierend auf dänischen Survey-Daten berichten Ejlskov et al. (2014) einen statistisch signifikanten Geschlechterunterschied bezüglich des Zusammenhangs von sozialem Kapital und Mortalität. Die Ergebnisse zeigen, auch nach Kontrolle von sozioökonomischem Status, Alter, Gesundheitsstatus und Gesundheitsverhalten, dass für Frauen* ein höheres Niveau an sozialem Kapital mit einem geringeren 
Mortalitätsrisiko (all-cause mortality) verbunden war. Ein weiterer theoretisch sehr bedeutsamer Befund der Studie ist der für Frauen* positive Zusammenhang zwischen der Kontakthäufigkeit mit Freund*innen und einem niedrigeren Sterblichkeitsrisiko. Auf negative Effekte des Sozialkapitals weisen auch Kawachi und Berkman (2001) hin. Demnach werden Frauen* durch ihr soziales Engagement mental stärker belastet und zeigen entsprechende Krankheitssymptome, wenn Personen mit denen sie verbunden sind, (gesundheitliche) Probleme bekommen. Daran anschließend berichten Sarason et al. (1997) und Antonucci et al. (1998), dass Frauen* stärker in soziale Beziehungen eingebunden sind und insbesondere, wenn sie über größere Netzwerke verfügen und viele enge Beziehungen pflegen, eher Stress und negative Effekte auf die allgemeine Lebenszufriedenheit erleben. Walen und Lachman (2000) zufolge mag dies daran liegen, dass Frauen*, die stärker in soziale Beziehungen eingebettet sind, auch eher negativen Ereignissen in ihrem sozialen Umfeld ausgesetzt sind (z. B. eine*n Freund*in bei Verlust einer geliebten Person unterstützen). Sie nehmen eher Hilfebedürfnisse anderer wahr, reagieren darauf und fungieren als Unterstützerinnen* in Krisen (Hobfoll und Vaux 1993; Nestmann und Schmerl 1992). Generell steht das Wohlbefinden der befragten Frauen* mehr mit positiven und negativen Aspekten der Ehe- und Freundschaftsbeziehungen in Verbindung als bei Männern* (Antonucci et al. 2001).

\subsection{Netzwerke und Geschlechterunterschiede mit Blick auf die Gesundheit}

Über diese Forschung zu sozialem Kapital hinausgehend, gibt es auch einige Studien, in denen eine dezidierte Netzwerkperspektive im Mittelpunkt steht und Geschlechterunterschiede eine wichtige oder zentrale Rolle einnehmen. Diese konzentrieren sich oft auf bestimmte Lebensphasen und dort insbesondere auf die netzwerkanalytisch schon vergleichsweise gut erforschte Jugendphase (siehe dazu auch den Beitrag: „Soziale Netzwerke, Gesundheit und gesundheitliche Ungleichheiten im Jugendalter") sowie auf die Phase des höheren Alters (siehe dazu auch den Beitrag: „Soziale Netzwerke und gesundheitliche Ungleichheiten im Alter"). Im Folgenden sollen einige neuere Befunde aus diesen Forschungsbereichen vorgestellt werden.

Ein wichtiges Thema im Jugendalter ist das Risikoverhalten, wie z. B. Tabakoder Alkoholkonsum. Hier finden sich sowohl Querschnitt- als auch Längsschnittstudien, die Geschlechterunterschiede beleuchten und sich dabei v. a. auf Netzwerke in Schulklassen fokussieren. Die Netzwerkforschung kann hier 
zeigen, dass spezifische Netzwerkeigenschaften, wie z. B. Homophilie, dafür sorgen, dass sich spezifisches Gesundheitsverhalten wie auch Interventionen zur Verbesserung des Gesundheitsverhaltens mehr oder weniger gut verbreiten können (Valente 2012).

So untersuchten beispielsweise Grard et al. (2018) in einer Querschnittsstudie Geschlechterunterschiede im Zigaretten-, Alkohol- und Cannabiskonsum bei 14bis 16-jährigen Jungen* und Mädchen* an 50 europäischen Schulen. Sie zeigen, dass Mädchen* eine geringere Prävalenz im Substanzkonsum haben als Jungen*. Aber auch das Geschlecht der Freund*innen spielt eine Rolle: Wenn Mädchen* in ihren Netzwerken mehr Freund*innen des anderen Geschlechts haben (other sex friendships, OSF), konsumieren sie eher eine der drei erhobenen Substanzen als Mädchen*, die mehr mit Mädchen* befreundet sind (same sex friendhips, SSF). Jungen* in OSF rauchen eher als Jungen* in SSF. Bei Alkohol- und Cannabiskonsum gehen bei Jungen* allerdings SSF eher mit dem Konsum dieser Substanzen einher. Auch die Geschlechterzusammensetzung an der Schule ist von Bedeutung: In Schulen, die männlich* dominiert sind, ist das Risiko des Substanzkonsums für Jungen* und Mädchen* höher.

Im Unterschied dazu finden Deutsch et al. (2014a) in ihrer Analyse basierend auf den Daten der National Longitudinal Study of Adolescent Health (Add Health) aus den USA ein Jahr später keinen Einfluss der Geschlechterzusammensetzung der Freundschaftsnetzwerke auf das Trinkverhalten. So bestätigt sich zwar die Hypothese der Autor*innen, dass der durchschnittliche Alkoholkonsum im peer-Netzwerk einen Einfluss auf den Alkoholkonsum von Ego hat, allerdings wird dies nicht vom Geschlecht (gender) moderiert. Die Autor*innen vermuten hier Selektionseffekte: Mädchen* suchen sich demnach peers, die ein ähnliches Trinkverhalten wie sie selbst aufweisen. Auch für das Geschlechterverhältnis in einer peer group konnte kein Einfluss auf den Alkoholkonsum von Ego nachgewiesen werden: Höhere Anteile von männlichen* Jugendlichen im Netzwerk führten entgegen der Annahme weder bei Jungen* noch bei Mädchen* zu einem höheren Alkoholkonsum. Überraschender Weise erwies sich allerdings die Nähe der Beziehungen als relevant auf den Alkoholkonsum: Sowohl bei Jungen* (SSF) als auch bei Mädchen* (OSF) ging eine geringere freundschaftliche Nähe zu männlichen* Freunden mit einem stärken Einfluss dieser Freunde auf den Alkoholkonsum ein Jahr später einher. Die Nähe zu Freundinnen* wurde auf diese Weise allerdings nur für Jungen* (OSF) bedeutsam. Die Autor*innen schließen aus ihren Befunden, dass die Rolle des Geschlechts bei der Sozialisation mit Alkohol viel komplexer ist als bisher angenommen, und fordern die Untersuchung einer Vielzahl von Beziehungen innerhalb eines Netzwerkes, auch solcher, die weniger eng oder nicht reziprok sind. Zudem müssten die Kontexte in 
denen Jugendliche trinken und ihre Motive für den Alkoholkonsum näher untersucht werden.

Den Effekt von Selektions- bzw. Einflussfaktoren, d. h. inwiefern sich Jugendliche ihre Peers nach ihren Vorlieben und Bedürfnissen auswählen oder durch diese in ihren Verhaltensweisen beeinflusst werden, gehen Studien mit Längsschnittdaten nach. Hierfür werden in vielen Fällen sogenannte SIENA-Modelle (Simulation Investigation for Empirical Network Analysis) verwendet. Die Forschungen fokussieren vor allem die Aspekte Alkohol-, Zigaretten und Cannabiskonsum bei Schüler*innen (z. B. Knecht et al. 2011; Osgood et al. 2013; Pearson et al. 2006). Hier sollen einige Beispielstudien angeführt werden: Bezüglich des Rauchverhaltens sind es bei finnischen Schüler*innen der Sekundärstufe eher Selektionsfaktoren die den Ausschlag für Freundschaftsbeziehungen geben. Beim Alkoholverhalten sind es sowohl Selektions- als auch Einflussfaktoren. Die Ergebnisse unterschieden sich nicht durchgängig in Bezug auf das Geschlecht (Kiuru et al. 2010). Auch Daw et al. (2015) können zeigen, dass Jungen* wie Mädchen* in den USA (7. Klasse) ihre gleichgeschlechtlichen Freund*innen nach Ähnlichkeit im Rauchverhalten auswählen. Ein Einfluss von Freundinnen* auf das Rauchverhalten konnte nur für Mädchen* nachgewiesen werden. Bezüglich des Alkoholkonsums stellen Burk et al. (2012) fest, dass die Ähnlichkeit zwischen dem Trinkverhalten von Freund*innen in der 6. Klasse beginnt, ihren Höhepunkt in der 8. Klasse erreicht und während der späten Adoleszenz wieder abnimmt. Jugendliche in allen drei Altersgruppen wählten Peers mit ähnlichem Trinkverhalten, wobei die Effekte bei frühjugendlichen Männern* und bei spätjugendlichen Frauen* am stärksten sind. Hinsichtlich des Einflusses gibt es keinen Unterschied zwischen den Geschlechtern (Burk et al. 2012). Bezüglich des Marihuanakonsums in High-Schools in den USA stellen die Autor*innen fest, dass auch der Freundeskreis nach Alter und Marihuanakonsum ausgesucht wird. Der Faktor Einfluss wurde nur an einer High-School festgestellt. Das Geschlecht, „race“ oder die Anzahl der Freund*innen außerhalb der Schule sagten jedoch nicht signifikant die Häufigkeit des Marihuanakonsums voraus. Ebenfalls gab es nur minimale Hinweise darauf, dass Peer-Effekte durch persönliche, schulische oder familiäre Risikofaktoren moderiert werden (De La Haye et al. 2013).

Für das Jugendalter lassen sich ferner Netzwerkstudien zu Geschlechterunterschieden und depressiven Erkrankungen finden. Ähnlich wie die Studie von Rosenquist et al. (2011) unter Erwachsenen, die zu dem Ergebnis kommt, dass Depressionen insbesondere für Frauen* sozial ansteckend sind, zeigen Conway et al. (2011) für das Jugendalter, dass bei Mädchen* das Auftreten von Depressionen im Freundeskreis mit einem verstärkten Auftreten eigener depressiver Symptome ein Jahr später einhergeht. 
Weitere Studien untersuchen sehr spezifische Netzwerkparameter und können zeigen, dass gleiche Netzwerkparameter für Mädchen* und Jungen* auf völlig unterschiedliche Weise mit depressiven Erkrankungen in Verbindung stehen. So leiden Jungen* eher an depressiven Erkrankungen, wenn sie Angst vor negativen Bewertungen durch ihre Peers erhalten und eine geringere Popularität in ihrem Netzwerk haben. Für Mädchen*, die Angst vor negativen Bewertungen haben, trifft dies eher zu, wenn sie eine hohe Popularität in ihren Netzwerken aufweisen (Kornienko und Santos 2014). Eine Studie von Falci und McNeely (2009) untersucht die Größe und Dichte von Netzwerken und zeigt, dass Mädchen*, wenn sie in sehr große, fragmentierte Netzwerke (d. h., nur wenige Netzwerkmitglieder kennen einander) eingebunden sind, eher von depressiven Symptomen betroffen sind als Mädchen*, die zwar in große aber kohäsive Netzwerke eingebunden sind. Bei Jungen* hingegen sind die Zusammenhänge genau umgekehrt: Sind sie in große und wenig kohäsive Netzwerke eingebunden, so sind sie weniger von depressiven Symptomen betroffen als Jungen*, die in große und kohäsive Netzwerke eingebettet sind.

Netzwerkstudien findet man auch in der Phase des hohen Alters. Eine Studie über ältere Menschen (über 60 Jahre) in den USA untersucht die Auswirkungen von unterschiedlichen idealtypischen Netzwerken (diverses Netzwerk, Netzwerk mit hohem gesellschaftlichem Engagement, Netzwerk mit geringem gesellschaftlichem Engagement und eingeschränkte Netzwerk) auf das Wohlbefinden. Männer*, die in eingeschränkte Netzwerke eingebunden sind, zeigen ein besonders niedriges Wohlbefinden. Im Allgemeinen bewerten Frauen*, in unterschiedlichen Netzwerktypen, ihre Gesundheit viel besser als Männer* (Fiori und Fuller 2017).

Ein wichtiges gesundheitsrelevantes Thema im höheren Alter ist auch der biografische Übergang der Verwitwung. Der Tod des*/der Partner*in kann negative Einflüsse auf die psychische Gesundheit haben und etwa zu depressiven Symptomen führen. Die Netzwerkmechanismen soziale Unterstützung, soziales Engagement und soziale Integration werden in diesem Zusammenhang als Faktoren genannt, die o. g. Symptome lindern und einen positiven Einfluss auf Gesundheit haben (vgl. den Beitrag: Soziale Netzwerke und gesundheitliche Ungleichheiten im Alter). Es gibt in diesem Zusammenhang einige Hinweise auf relevante geschlechtsspezifische Unterschiede (vgl. Monserud und Wong 2015): Ältere Männer* verlassen sich eher auf ihre Frauen*, wenn es um emotionale Unterstützung, die Haushaltsführung und das Pflegen sozialer Kontakte geht (siehe auch Lee et al. 2001; Umberson et al. 1992) und Frauen* befinden sich eher in ökonomischer Abhängigkeit von ihrem Mann* und können daher bei Verwitwung finanziellem Stress ausgesetzt sein (Arber 2004; Umberson et al. 1992). Zudem steht ausschließlich bei älteren Frauen* eine als gering wahrgenommene 
soziale Unterstützung und nur bei älteren Männern* eine geringere Netzwerkeinbindung in Beziehung zu einer schlechteren selbstberichteten Gesundheit (Caetano et al. 2013). Hieraus könnten sich unterschiedliche Anforderungen an die sozialen Beziehungsnetzwerke ergeben, die diese nicht immer erfüllen können.

Für Mexiko, ein Land, in dem institutionelle Hilfesysteme weniger ausgebaut und private, familiäre Unterstützungsstrukturen daher wichtiger sind, finden Monserud und Wong (2015) in einer Längsschnittstudie etwa, dass verheiratete Männer* weniger depressive Symptome angaben als alle anderen nach Geschlecht differenzierten Statusgruppen (verheiratet/bereits in Welle 1 verwitwet/in Welle 2 verwitwet). Allerdings gab es bei den kürzlich (seit Welle 2) verwitweten Personen keine statistisch signifikanten Geschlechterunterschiede bezüglich depressiver Symptomatiken. Die Ergebnisse zum Einfluss sozialer Unterstützung sind uneinheitlich bzw. die Effekte müssen differenziert betrachtet werden: Unabhängig vom Familienstand hängt ein höherer Wert bei emotionaler Unterstützung mit niedrigeren Zunahmen depressiver Symptome zusammen, während der Empfang finanzieller oder praktischer Unterstützung - bei kürzlich verwitweten Männern* ausgeprägter als bei kürzlich verwitweten Frauen* - mit einer stärkeren Zunahme dieser Symptome verbunden ist. Dies könnte damit zusammenhängen, dass die Angewiesenheit auf diese Form der Unterstützung Gefühle der Abhängigkeit auslösen, mit der Wahrnehmung einer eingeschränkten Autonomie und einer Umkehr der Rollen in den Eltern-Kind-Beziehungen verbunden sein könnte und damit Stress hervorruft (siehe dazu auch den Beitrag „Negative Beziehungsaspekte und gesundheitliche Ungleichheiten“). Eine stärkere Integration in ein soziales Netzwerk, die über Ko-Residenz mit Kindern, Verwandten oder Freund*innen sowie der Teilnahme an Gemeinschaftsaktivitäten operationalisiert wurde, hat ebensolche differenziert $\mathrm{zu}$ betrachtenden Effekte: Allgemein ist Ko-Residenz mit Verwandten mit einer höheren Zunahme depressiver Symptome verbunden, während Ko-Residenz mit anderen (Kindern, Freund*innen) eine geringere Zunahme depressiver Symptome bedeutet. Bei kürzlich verwitweten Männern* und bei seit längerem verwitweten Frauen* hängt Ko-Residenz mit Kindern mit einer geringeren Zunahme depressiver Symptome zusammen, während für kürzlich verwitwete Männer* die Ko-Residenz mit anderen Personen mit einer stärkeren Zunahme einhergeht. Soziale Integration in Gemeinschaftsaktivitäten liefert allgemein keinen Erklärungsbeitrag für die Veränderung bei depressiven Symptomen zwischen den beiden Wellen. Bei kürzlich verwitweten Frauen* hängt der Kirchenbesuch mit einem stärkeren Anstieg zusammen, während freiwillige Arbeit in Gemeinschaftsaktivitäten bei seit längerem verwitweten Frauen* mit einem niedrigeren Anstieg assoziiert ist. Es gibt also deutliche Hinweise darauf, dass soziale Unterstützung und soziale 
Integration für die Geschlechter eine unterschiedliche Bedeutung haben und dass hierbei Rollenbilder und eine ungleiche Verteilung von Aufgaben im Haushalt und der Partnerschaft eine Rolle spielen. Nach der Theorie des Sozialkapitals stellen soziale Netzwerke auch für ältere Trans*-Personen ein Vehikel für soziale Ressourcen dar, die für ein erfolgreiches Altern und Wohlbefinden von Vorteil sein können: „Controlling for background characteristics, network size was positively associated with being female, transgender identity, employment, higher income, having a partner or a child, identity disclosure to a neighbor, engagement in religious activities, and service use. Controlling in addition for network size, network diversity was positively associated with younger age, being female, transgender identity, identity disclosure to a friend, religious activity, and service use“" (Erosheva et al. 2016, S. 98).

\section{$4 \quad$ Fazit}

Zusammenfassend lässt sich sagen, dass die Kategorie Geschlecht, im Vergleich $\mathrm{zu}$ den anderen vorgestellten Kategorien in diesem Buch, relativ gut untersucht sind. Dennoch wird der Begriff des Netzwerkes häufig als Metapher anstatt einer Methode oder Theorie verwendet. Der Fokus liegt vor allem auf Schulklassenstudien und älteren Menschen.

Studien weisen u. a. darauf hin, dass Frauen* länger leben als Männer*. Auch bezüglich der Morbidität existieren gesundheitliche Unterschiede zwischen den Geschlechtern. Besonders im Jugendalter schneiden Jungen* in den meisten gesundheitsbedingten Indikatoren (z. B. Leukämie, Epilepsie, chronische Krankheiten) schlechter ab als Mädchen*. In der Pubertät scheinen Mädchen* eher an psychosomatischen und physiologischen Beschwerden zu leiden. Das weibliche* Krankheitsprofil ist ab diesen Zeitpunkt eher durch chronische Erkrankungen sowie durch psychosomatische und psychische Beeinträchtigungen (z. B. Schilddrüsenerkrankungen, Depression, Essstörungen) gekennzeichnet; das männliche* hingegen durch akute und lebensbedrohliche Krankheiten (z. B. HIV-Infektion, bösartige Neubildungen der Verdauungsorgane sowie der Lungen und Bronchien). Im hohen Alter ist kaum ein genereller Geschlechterunterschied zu erkennen. Hinsichtlich des Risikoverhaltens zeigen Forschungen, dass Männer* sowohl mehr rauchen als auch mehr Alkohol zu sich nehmen als Frauen*. Während bis ins Erwachsenenalter hinein Männer* seltener psychiatrische und psychotherapeutische sowie ambulante ärztliche Leistungen in Anspruch nehmen, gleicht sich dies im hohem Alter wieder an. 
Netzwerkstudien stellen Differenzen zwischen Männern* und Frauen* fest. Es kann behauptet werden, dass Frauen* größere Netzwerke besitzen, die ihrerseits mehr familiäre und verwandtschaftliche Diversität aufweisen. Neuere Studien gehen jedoch davon aus, dass sich die Netzwerke beider Geschlechter langsam angleichen. Mit Blick auf die aus den sozialen Beziehungen gewonnenen Ressourcen finden sich Belege, dass Hilfe im Krankheitsfall häufiger von Frauen* geleistet wird. Mütter* übernehmen auch den zeitintensiveren Support, und Frauen* scheinen mehr Ansprechpartner*innen für Probleme zu besitzen als Männer*. Wer bei der Hilfeinanspruchnahme bevorzugt wird, Männer* oder Frauen*, scheint hingegen widersprüchlich, wobei sich in mehr Studien ein Hang zu weiblichen* Helferinnen zeigt. Bei beruflichen Netzwerkbeziehungen zeigt sich folgendes Bild: Männer* haben größere arbeitsbezogene Netzwerke, sie sind anderen Subnetzwerken verbunden und ziehen mehr Vorteile aus diesen Beziehungen, da Männer* höhere Positionen in beruflichen Netzwerken einnehmen. Frauen* scheinen sich eher auf ihre individuellen Kompetenzen statt auf das Sozialkapital zu fokussieren, während Männer* stärker auf Netzwerke setzen und die Ressourcen besser nutzen.

Studien über den Zusammenhang von Netzwerken und Sozialkapital oder sozialer Unterstützung vor dem Hintergrund gesundheitlicher Ungleichheiten zeigen eine ungleiche Verteilung zwischen den Geschlechtern. Frauen* scheinen mehr und zeitaufwendigere soziale Unterstützungsaufgaben zu übernehmen. Sie besitzen mehr Ansprechpartner*innen für Probleme als Männer*. Daneben leiden sie wohl auch häufiger unter negativen Aspekten von sozialen Beziehungen. Es wird davon ausgegangen, dass Frauen* durch ihr stärkeres soziales Engagement höheren gesundheitlichen Belastungen ausgesetzt sind als Männer*.

Im Allgemeinen rücken die Gesundheit sowie das Gesundheitsverhalten von Schüler*innen und älteren Menschen in den Fokus der Netzwerkforschung. Bei Jugendlichen und Heranwachsenden untersuchen Netzwerkstudien häufig den Zigaretten-, Alkohol- und Cannabiskonsums. Neben den Querschnittstudien gehen neuere Längsschnittstudien den Einfluss- bzw. Selektionsfaktoren nach. Sie untersuchen die Frage, inwieweit sich Jugendliche ihre Freund*innen nach ihren Vorlieben und Bedürfnissen auswählen oder durch diese in ihren Verhaltensweisen beeinflusst werden. Hier scheint die Forschungslage, vielleicht auch aufgrund der unterschiedlichen Datensätze und Länderfokussierung, doch eher heterogen. Es wird allerdings deutlich, dass Mädchen* oder junge Frauen* im geringeren Maße leichte Drogen zu sich nehmen als ihre männlichen* Altersgenossen und soziale Netzwerke einen großen Effekt auf das Gesundheitsverhalten besitzen. Inwieweit es bei den Netzwerkeffekten Geschlechtsunterschiede 
gibt, bleibt jedoch noch offen. Auch zwischen depressiven Erkrankungen und sozialen Netzwerken zeigt sich ein Zusammenhang, der allem Anschein nach geschlechtsspezifischen Faktoren unterliegt.

Im Alter stehen vor allem die Phase der Verwitwung und die damit zusammenhängenden Netzwerkeffekte im Blickpunkt. Es scheint so zu sein, dass Netzwerke einen positiven Einfluss auf Gesundheit besitzen. Dennoch zeigen sich auch negative Seiten von Netzwerken. Hier gibt es Hinweise auf Geschlechtsunterschiede. Beispielsweise verlieren Männer* durch den Tod ihrer Frau* eher emotionale Unterstützung und Teile der sozialen Kontakte, während Frauen* durch die ökonomische Abhängigkeit von ihrem Mann* finanziellem Stress ausgesetzt sein können.

Die sehr wenigen Studien zu Trans*-Personen zeigen, dass viele Trans*Personen am Rande der Gesellschaft leben und mit Stigmatisierung, Diskriminierung, Ausgrenzung, Gewalt und schlechter Gesundheit konfrontiert sind (Winter et al. 2016).

Als Fazit möchten wir noch kurz auf die Desiderata eingehen. Trotz einer Anzahl an wissenschaftlichen Untersuchungen sind bisher noch viele Fragen ungeklärt. Vor allem wollen wir darauf hinweisen, dass intergeschlechtliche Personen $^{11}$ in den Studien (fast) nie Berücksichtigung finden. Ferner spielt bei der Analyse zum Nutzen von sozialer Unterstützung oder Sozialkapital Netzwerkforschung bisher eine untergeordnete Rolle. Aber auch konkrete Fragen bleiben kaum berücksichtigt. Während bei Risikoverhalten der Einfluss von Netzwerken bereits sehr gut erforscht ist, stellt sich die Frage, welche positiven Aspekte soziale Netzwerke auf das Gesundheitsverhalten wie z. B. Sporttreiben oder auch das Entsagen von bestimmten Drogen haben. Auch bei den Erklärungsmustern hinsichtlich der Erkrankung und dem Verlauf von Krankheiten, sollte die Frage nach den Effekten von Schicht, Geschlecht und sozialen Netzwerkverbindungen noch stärker in den Blickpunkt genommen werden und dies nicht nur mit neueren Verfahren der quantitativen sondern auch der qualitativen Netzwerkforschung. Zudem wäre es hier wichtig, das Konzept der Intersektionalität noch besser mit der Netzwerkforschung zu verbinden.

\footnotetext{
${ }^{11}$ Menschen, deren körperliche Merkmale sich nicht der weiblichen oder männlichen Geschlechternorm zuordnen lassen (Gerede e. V. 2018).
} 


\section{Leseempfehlungen}

Kolip, P. \& Hurrelmann, K. (2016). Handbuch Geschlecht und Gesundheit: Männer und Frauen im Vergleich. Bern: Hogrefe. Das Handbuch bietet einen aktuellen und umfassenden Überblick über den wissenschaftlichen Forschungsstand und interdisziplinäre Diskurse im Kontext von Geschlecht und Gesundheit.

Barry, A.-M. \& Yuill, C. (2012). Understanding the sociology of health: An introduction. 3. Aufl. Los Angeles, CA: Sage. Der Abschnitt zu Gender and Health (S. 129-144) bietet den Leser*innen eine gut verständliche und auf internationalen Daten basierende Einführung in die Zusammenhänge von Gesundheit und Geschlecht.

Bradford, J., Reisner, S. L., Honnold J. A. \& Xavier, J. (2013). Lesbian, gay, bisexual, and transgender health: Findings and concerns. Journal of the Gay and Lesbian Medical Association, 4 (3), S. 102-151. Ein dichter und guter englischer Überblicksartikel zum Thema LGBT und Gesundheit.

Moore, G. (1990). Structural determinants of men's and women's personal networks. American Sociological Review, 55 (5), S. 726-735. Ältere, aber beispielhafte repräsentative Studie aus den USA, die mit quantitativen Daten des General Social Survey (GSS, 1985) zu starken Beziehungen arbeitet.

Schwartz, E. \& Litwin, H. (2018). Social network changes among older Europeans: The role of gender. European Journal of Ageing, 15 (4), S. 359-367. Aktuelle, quantitative Längsschnittstudie, die mithilfe des europaweit angelegten Survey of Health, Ageing, and Retirement in Europe $(\mathrm{n}=13,938)$ nach Geschlechterdifferenzen in den sozialen Netzwerken älterer Menschen (65+) fragt.

\section{Literatur}

Antonucci, T. C., Akiyama, H., \& Lansford, J. E. (1998). Negative effects of close social relations. Family Relations, 47(4), 379-384.

Arber, S. (2004). Gender, marital status, and ageing: Linking material, health, and social resources. Journal of Aging Studies, 18(1), 91-108.

Babitsch, B. (2009). Die Kategorie Geschlecht: Theoretische und empirische Implikationen für den Zusammenhang zwischen sozialer Ungleichheit und Gesundheit. In M. Richter \& K. Hurrelmann (Hrsg.), Gesundheitliche Ungleichheit. Grundlagen, Probleme, Perspektiven (2., aktualisierte Aufl., S. 283-299). Wiesbaden: VS. 
Barker, C., Pistrang, N., Shapiro, D. A., \& Shaw, I. (1990). Copig and help-seeking in the UK adult population. British Journal of Clinical Psychology, 29(3), 271-285.

Barry, A.-M., \& Yuill, C. (2012). Understanding the sociology of health. An introduction (3. Aufl.). Los Angeles: SAGE.

Bartley, M. (2017). Health inequality. An introduction to concepts, theories and methods (2. Aufl.). Cambridge: Polity.

Bearman, P. S., Moody, J., \& Stovel, K. (2004). Chains of affection: The structure of adolescent romantic and sexual networks. American Journal of Sociology, 110(1), 44-91.

Bearman, P., \& Parigi, P. (2004). Cloning headless frogs and other important matters: Conversation topics and network structure. Social Forces, 83(2), 535-557.

Benotsch, E. G., Zimmerman, R., Cathers, L., McNulty, S., Pierce, J., Heck, T., \& Snipes, D. (2013). Non-medical use of prescription drugs, polysubstance use, and mental health in transgender adults. Drug and Alcohol Dependence, 132(1-2), 391-394.

Bott, E. (1957). Family and social network: Roles, norms and external relationships in ordinary urban families. London: Tavistock.

Bradford, J., Reisner, S. L., Honnold, J. A., \& Xavier, J. (2013). Lesbian, gay, bisexual, and transgender health: Findings and concerns. Journal of the Gay and Lesbian Medical Association, 4(3), 102-151.

Bundesversicherungsamt. (2017). Tätigkeitsbericht 2017. https://www.bundesversicherungsamt.de/fileadmin/redaktion/Presse/epaper2017/index.html\#0. Zugegriffen: 28. März 2019.

Burk, W. J., Van Der Vorst, H., Kerr, M., \& Stattin, H. (2012). Alcohol use and friendship dynamics: Selection and socialization in early-, middle-, and late-adolescent peer networks. Journal of Studies on Alcohol and Drugs, 73(1), 89-98.

Caetano, S. C., Silva, C. M. F. P., \& Vettore, M. V. (2013). Gender differences in the association of perceived social support and social network with self-rated health status among older adults: A population-based study in Brazil. BMC Geriatrics, 13, 122.

Chua, V., Mathews, M., \& Loh, Y. C. (2016). Social capital in Singapore: Gender differences, ethnic hierarchies, and their intersection. Social Networks, 47, 138-150.

Conway, C. C., Rancourt, D., Adelman, C. B., Burk, W. J., \& Prinstein, M. J. (2011). Depression socialization within friendship groups at the transition to adolescence: The roles of gender and group centrality as moderators of peer influence. Journal of Abnormal Psychology, 120(4), 857-867.

Daw, J., Margolis, R., \& Verdery, A. M. (2015). Siblings, friends, course-mates, club-mates: How adolescent health behavior homophily varies by race, class, gender, and health status. Social science \& medicine, 1982(125), 32-39.

De La Haye, K., Green, H. D., Kennedy, D. P., Pollard, M. S., \& Tucker, J. S. (2013). Selection and influence mechanisms associated with marijuana initiation and use in adolescent friendship networks. Journal of Research on Adolescence, 23(3), 474-486.

Dean, L., Meyer, I. H., Robinson, K., Sell, R. L., Sember, R., Silenzio, V. M., \& Dunn, P. (2000). Lesbian, gay, bisexual, and transgender health: Findings and concerns. Journal of the Gay and Lesbian Medical Association, 4(3), 102-151.

Degele, N. (2008). Gender/Queer Studies. Eine Einführung. Paderborn: Wilhelm Fink.

Deutsch, A. R., Steinley, D., \& Slutske, W. S. (2014). The role of gender and friends' gender on peer socialization of adolescent drinking: A prospective multilevel social network analysis. Journal of Youth and Adolescence, 43(9), 1421-1435. 
Diewald, M. (1991). Soziale Beziehungen: Verlust oder Liberalisierung? Soziale Unterstützung in informellen Netzwerken. Berlin: Edition Sigma.

Döring, N. (2013). Zur Operationalisierung von Geschlecht im Fragebogen: Probleme und Lösungsansätze aus Sicht von Mess-, Umfrage-,Gender- und Queer-Theorie. Gender, 5(2), 94-113.

Dorsen, C. (2012). An integrative review of nurse attitudes towards lesbian, gay, bisexual, and transgender patients. Canadian Journal of Nursing Research, 44(3), 18-43.

Ejlskov, L., Mortensen, R. N.; Overgaard, C., Christensen, L. R. B. U., Vardinghus-Nielsen, H., Kræmer, S. R. J., Wissenberg, M., ... Hansen, C. D. (2014). Individual social capital and survival: A population study with 5-year follow-up. BMC Public Health, 14(1025).

Erosheva, E. A., Kim, H. J., Emlet, C., \& Fredriksen-Goldsen, K. I. (2016). Social networks of lesbian, gay, bisexual, and transgender older adults. Research on Aging, 38(1), 98-123.

Falci, C. D., \& McNeely, C. (2009). Too many friends: Social integration, network cohesion and adolescent depressive symptoms. Social Forces, 87(4), 2031-2062.

Feiring, C. (1999). Other-sex friendship networks and the development of romantic relationships in adolescence. Journal of Youth and Adolescence, 28(4), 495-512.

Fiori, K. L., \& Fuller, H. R. (2017). Social network typologies and health in late life: The moderating role of gender. Innovation in Aging, 1(Suppl. 1), 941.

Fischer, C. S., \& Oliker, S. J. (1983). A research note on friendship, gender, and the life cycle. Social Forces, 62, 123-133.

Fischer, C. S. (1982). To dwell among friends: Personal networks in town and city. Chicago, IL: University of Chicago Press.

Fischer, C. S. (2009). The 2004 GSS finding of shrunken social networks: An artifact? American Sociological Review, 74(4), 657-669.

Fuller-Iglesias, H. R., \& Antonucci, T. C. (2016). Familism, social network characteristics, and well-being among older adults in Mexico. Journal of Cross-Cultural Gerontology, 31(1), 1-17.

Gerede e. V. (2018). Trans* - Eine Informationsbroschüre zum Thema Transidentität für betroffene Menschen, Angehörige und Interessierte. https://gerede-dresden.de/index. php/infomaterial.html. Zugegriffen: 13. Mai 2019.

Gillespie, B. J., Lever, J., Frederick, D., \& Royce, T. (2015). Close adult friendships, gender, and the life cycle. Journal of Social and Personal Relationships, 32(6), 709-736.

Graham, R., Berkowitz, B., Blum, R., Bockting, W., Bradford, J., de Vries, B., \& Makadon, H. (2011). The health of lesbian, gay, bisexual, and transgender people: Building a foundation for better understanding. Washington, DC: Institute of Medicine.

Grant, J. M., Mottet, L., Tanis, J. E., Harrison, J., Herman, J., \& Keisling, M. (2011). Injustice at every turn: A report of the national transgender discrimination survey. Washington, DC: National Center for Transgender Equality.

Grard, A., Kunst, A., Kuipers, M., Richter, M., Rimpela, A., Federico, B., \& Lorant, V. (2018). Same-sex friendship, school gender composition, and substance use: A social network study of 50 European schools. Substance Use \& Misuse, 53(6), 998-1007.

Grunow, D., Breitkopf, H., Dahme, H.-J., Engfer, R., Grunow-Lutter, V., \& Paulus, W. (1983). Gesundheitsselbsthilfe im Alltag. Ergebnisse einer repräsentativen Haushaltsbefragung über gesundheitsbezogene Selbsterfahrungen und -potentiale. Stuttgart: Ferdinand Enke. 
Haas, A. P., Eliason, M., Mays, V. M., Mathy, R. M., Cochran, S. D., D’Augelli, A. R., Silverman..., M. M., \& Clayton, P. J. (2010). Suicide and suicide risk in lesbian, gay, bisexual, and transgender populations: Review and recommendations. Journal of Homosexuality, 58(1), 10-51.

Hapke, U., Maske, U. E., Scheidt-Nave, C., Bode, L., Schlack, R., \& Busch, M. (2013). Chronischer Stress bei Erwachsenen in Deutschland. Ergebnisse der Studie zur Gesundheit Erwachsener in Deutschland (DEGS1). Bundesgesundheitsblatt, 56(5/6), 749-775.

Herbst, J. H., Jacobs, E. D., Finlayson, T. J., McKleroy, V. S., Neumann, M. S., Crepaz, N., \& HIV/AIDS Prevention Research Synthesis Team. (2008). Estimating HIV prevalence and risk behaviors of transgender persons in the United States: A systematic review. AIDS and Behavior, 12(1), 1-17.

Hobfoll, S. E., \& Vaux, A. (1993). Social support: Social resources and social context. In L Goldberg \& S. Breznitz (Hrsg.), Handbook of stress. Theoretical and clinical aspects (2. Aufl., S. 685-705). New York: Free Press.

House, J. S., Umberson, D., \& Landis, K. R. (1988). Structures and processes of social support. Annual Review of Sociology, 14(1), 293-318.

Hurrelmann, K., \& Quenzel, G. (2011). Geschlecht und Gesundheit. Public Health Forum, 19(2), 2-3.

Iller, C., \& Wienberg, J. (2012). Altern und Geschlecht. Gesundheit und Wohlbefinden im Alter in einer geschlechterdifferenziellen Perspektive. In V. Moser \& B. Rendtorff (Hrsg.), Riskante Leben? Geschlechterordnungen in der Reflexiven Moderne (S. 83-92). Opladen: Budrich.

i-Päd - Initiative intersektionale Pädagogik. (2019). Glossar. http://www.i-paed-berlin.de/ de/Glossar/\#queer. Zugegriffen: 13. Mai 2019.

Kawachi, I., \& Berkman, L. F. (2001). Social ties and mental health. Journal of Urban Health, 78(3), 458-467.

Kecojevic, A., Wong, C. F., Schrager, S. M., Silva, K., Bloom, J. J., Iverson, E., \& Lankenau, S. E. (2012). Initiation into prescription drug misuse: Differences between lesbian, gay, bisexual, transgender (LGBT) and heterosexual high-risk young adults in Los Angeles and New York. Addictive Behaviors, 37(11), 1289-1293.

Kiuru, N., Burk, W. J., Laursen, B., Salmela-Aro, K., \& Nurmi, J. E. (2010). Pressure to drink but not to smoke: Disentangling selection and socialization in adolescent peer networks and peer groups. Journal of Adolescence, 33(6), 801-812.

Knecht, A. B., Burk, W. J., Weesie, J., \& Steglich, C. (2011). Friendship and alcohol use in early adolescence: A multilevel social network approach. Journal of Research on Adolescence, 21(2), 475-487.

Kolip, P., \& Hurrelmann, K. (2002). Geschlecht-Gesundheit-Krankheit: Eine Einführung. In: Hurrelmann, K. \& Kolip, P. (Hrsg.). Geschlecht, Gesundheit und Krankheit. Männer und Frauen im Vergleich. 1. Aufl. Bern, Göttingen, Toronto, Seattle: Huber (Verlag Hans Huber, Programmbereich Gesundheit), S. 13-31.

Kolip, P., \& Hurrelmann, K. (2016). Geschlecht und Gesundheit: eine Einführung. In P. Kolip \& K. Hurrelmann (Hrsg.), Handbuch Geschlecht und Gesundheit. Männer und Frauen im Vergleich (2. Aufl., S. 8-18). Bern: Hogrefe.

Kornienko, O., \& Santos, C. (2014). The effects of friendship network popularity on depressive symptoms during early adolescence: Moderation by fear of negative evaluation and gender. Journal of Youth and Adolescence, 43(4), 541-553. 
Kuhlmann, E. (2016). Gendersensible Perspektiven auf Gesundheit und Gesundheitsversorgung. In M. Richter \& K. Hurrelmann (Hrsg.), Soziologie von Gesundheit und Krankheit (S. 183-196). Wiesbaden: Springer VS.

Lampert, T., Hoebel, J., Kuntz, B., Müters, S., \& Kroll, L. E. (2017), Gesundheitliche Ungleichheit in verschiedenen Lebensphasen. Gesundheitsberichterstattung des Bundes. https://www.rki.de/DE/Content/Gesundheitsmonitoring/Gesundheitsberichterstattung/GBEDownloadsB/gesundheitliche_ungleichheit_lebensphasen.pdf. Zugegriffen: 20. März 2019.

Lange, C., Manz, K., \& Kuntz, B. (2017). Alkoholkonsum bei Erwachsenen in Deutschland: Riskante Trinkmengen. https://www.rki.de/DE/Content/Gesundheitsmonitoring/ Gesundheitsberichterstattung/GBEDownloadsJ/FactSheets/JoHM_2017_02_Alkoholkonsum_Erwachsene.pdf. Zugegriffen: 20. März 2019.

Lee, G. R., DeMaris, A., Bavin, S., \& Sullivan, R. (2001). Gender differences in the depressive effect of widowhood in later life. The Journals of Gerontology: Series B, 56(1), S56-S61.

Lenz, K. (2003). Zur Geschlechtstypik persönlicher Beziehungen - Eine Einführung. In K. Lenz (Hrsg.), Frauen und Männer. Zur Geschlechtstypik persönlicher Beziehungen (S. 7-51). Weinheim: Juventa.

Lepore, S. J. (1992). Social conflict, social support, and psychological distress: Evidence of cross-domain buffering effects. Journal of Personality and Social Psychology, 63(5), $857-867$.

Lin, N. (2000). Inequality in social capital. Contemporary Sociology, 29(6), 785-795.

Lubbers, M. J., \& Snijders, T. A. (2007). A comparison of various approaches to the exponential random graph model: A reanalysis of 102 student networks in school classes. Social Networks, 29(4), 489-507.

Lyness, K. S., \& Thompson, D. E. (2000). Climbing the corporate ladder: Do female and male executives follow the same route? Journal of Applied Psychology, 85(1), 86-101.

Marsden, P. V. (1987). Core discussion networks of Americans. American Sociological Review, 52(1), 122-131.

Martin, C. L., Kornienko, O., Schaefer, D. R., Hanish, L. D., Fabes, R. A., \& Goble, P. (2013). The role of sex of peers and gender-typed activities in young children's peer affiliative networks: A longitudinal analysis of selection and influence. Child Development, 84(3), 921-937.

McGuire, G. M. (2000). Gender, race, ethnicity, and networks: The factors affecting the status of employees' network members. Work and Occupations, 27(4), 501-524.

McPherson, M., Smith-Lovin, L., \& Brashears, M. E. (2006). Social isolation in America: Changes in core discussion networks over two decades. American Sociological Review, 71(3), 353-375.

McPherson, M., Smith-Lovin, L., \& Cook, J. M. (2001). Birds of a feather: Homophily in social networks. Annual Review of Sociology, 27(1), 415-444.

Mercken, L., Snijders, T. A. B., Steglich, C., Vertiainen, E., \& de Vries, H. (2010). Smoking-based selection and influence in gender-segregated friendship networks: A social network analysis of adolescent smoking. Addiction, 105(7), 1280-1289.

Miller, J. B. (1976). Toward a new psychology of women. Boston: Beacon.

Monserud, M. A., \& Wong, R. (2015). Depressive symptoms among older Mexicans: The role of widowhood, gender, and social integration. Research on Aging, 37(8), 856-886.

Moore, G. (1990). Structural determinants of men's and women's personal networks. American Sociological Review, 55(5), 726-735. 
Munch, A., McPherson, J. M., \& Smith-Lovin, L. (1997). Gender, children, and social contact: The effects of childrearing for men and women. American Sociological Review, 62(2), 509-520.

Mustanski, B. S., Garofalo, R., \& Emerson, E. M. (2010). Mental health disorders, psychological distress, and suicidality in a diverse sample of lesbian, gay, bisexual, and transgender youths. American Journal of Public Health, 100(12), 2426-2432.

Nestmann, F., \& Schmerl, C. (1992). Wer hilft im Alltag? Gruppendynamik, 23(2), 161-179.

Newcomb, M. E., Heinz, A. J., \& Mustanski, B. (2012). Examining risk and protective factors for alcohol use in lesbian, gay, bisexual, and transgender youth: A longitudinal multilevel analysis. Journal of Studies on Alcohol and Drugs, 73(5), 783-793.

Nieder, T.O., Briken, P., \& Güldenring, A. K. (2016). Eine aktuelle Übersicht zur Diagnostik und Behandlung. Geschlechtsinkongruenz, -dysphorie und Trans*-Gesundheit. InFo Neurologie und Psychatrie, 18(12), 38-49.

Oehlendieck, L. (2003). Gender Trouble in Organisationen und Netzwerken. In U. Pasero \& C. Weinbach (Hrsg.), Frauen, Männer, Gender Trouble: Systemtheoretische Essays (S. 171-185). Frankfurt a. M.: Suhrkamp.

Orth, B., \& Töppich, J. (2015). Der Alkoholkonsum Jugendlicher und junger Erwachsener in Deutschland 2014. Ergebnisse einer aktuellen Repräsentativbefragung und Trends. Köln: Bundeszentrale für gesundheitliche Aufklärung.

Osgood, D. W., Ragan, D. T., Wallace, L., Gest, S. D., Feinberg, M. E., \& Moody, J. (2013). Peers and the emergence of alcohol use: Influence and selection processes in adolescent friendship networks. Journal of Research on Adolescence, 23(3), 500-512.

Pabst, A., Kraus, L., Gomes de Matos, E., \& Piontek, D. (2013). Substanzkonsum und substanzbezogene Störungen in Deutschland im Jahr 2012. Sucht, 59(6), 321-331.

Paik, A., \& Sanchagrin, K. (2013). Social isolation in America: An artifact. American Sociological Review, 78(3), 339-360.

Pearson, M., Steglich, C., \& Snijders, T. (2006). Homophily and assimilation among sport-active adolescent substance users. Connections, 27(1), 47-63.

Pflum, S. R., Testa, R. J., Balsam, K. F., Goldblum, P. B., \& Bongar, B. (2015). Social support, trans community connectedness, and mental health symptoms among transgender and gender nonconforming adults. Psychology of Sexual Orientation and Gender Diversity, 2(3), 281-286.

Poole, M., \& Bornholt, L. (1998). Career development of academics: Cross-cultural and lifespan factors. International Journal of Behavioral Development, 22(1), 103-126.

Poteat, T., German, D., \& Kerrigan, D. (2013). Managing uncertainty: A grounded theory of stigma in transgender health care encounters. Social Science \& Medicine, 84, 22-29.

Prütz, F., \& Rommel, A. (2017). Inanspruchnahme von Krankenhausbehandlungen in Deutschland. https://www.rki.de/DE/Content/Gesundheitsmonitoring/Gesundheitsberichterstattung/GBEDownloadsJ/FactSheets/JoHM_04_2017_Krankenhausbehandlungen.pdf. Zugegriffen: 20. März 2019.

Regitz-Zagrosek, V. (2017). Geschlecht und Herz-Kreislauf-Erkrankungen: Warum wir Gendermedizin brauchen. Der Internist, 58(4), 336-343.

Regitz-Zagrosek, V. (2018). Gesundheit, Krankheit und Geschlecht. Aus Politik und Zeitgeschichte, 68(24), 19-24.

Robert-Koch-Institut (2015). Gesundheit in Deutschland. Gesundheitsberichterstattung des Bundes. http://www.gbe-bund.de/pdf/GESBER2015.pdf. Zugegriffen: 25. März 2019. 
Rommel, A., \& Prütz, F. (2017). Inanspruchnahme physiotherapeutischer Leistungen in Deutschland. https://www.rki.de/DE/Content/Gesundheitsmonitoring/Gesundheitsberichterstattung/GBEDownloadsJ/FactSheets/JoHM_04_2017_Physiotherapeutische_Leistungen.pdf?_blob=publicationFile. Zugegriffen: 20.03.2019.

Rose, L. (2015). Geschlecht als soziale Unterscheidungskategorie in unserer Lebenswelt. In B. Bretländer, M. Köttig, \& T. Kunz (Hrsg.), Vielfalt und Differenz in der sozialen Arbeit. Perspektiven auf Inklusion (S. 63-73). Stuttgart: W. Kohlhammer.

Rosenquist, J. N., Fowler, J. H., \& Christakis, N. A. (2011). Social network determinants of depression. Molecular Psychiatry, 16(3), 273-281.

Rowe, C., Santos, G. M., McFarland, W., \& Wilson, E. C. (2015). Prevalence and correlates of substance use among trans* female youth ages 16-24 years in the San Francisco Bay Area. Drug and Alcohol Dependence, 147, 160-166.

Sarason, B. R., Sarason, I. G., \& Gurung, R. A. R. (1997). Close personal relationships and health outcomes: A key to the role of social support. In S. Duck (Hrsg.), Handbook of personal relationships: Theory, research and interventions (S. 547-573). Hoboken: Wiley.

Schmid, T. (2014). Generation, Geschlecht und Wohlfahrtsstaat. Intergenerationelle Unterstützung in Europa. Wiesbaden: Springer VS.

Schwartz, E., \& Litwin, H. (2018). Social network changes among older Europeans: The role of gender. European Journal of Ageing, 15(4), 359-367.

Sheridan, A. (2002). What you know and who you know: "Successful" women's experiences of accessing board positions. Career Development International, 7(4), 203-210.

Sieverding, M. (2005). Geschlecht und Gesundheit. In R. Schwarzer \& N. (Hrsg.), Gesundheitspsychologie (S. 55-70). Göttingen: Hogrefe.

Statistisches Bundesamt (2019a). Genesis-Online-Datenbank. https://www-genesis.destatis. de/genesis/online. Zugegriffen: 22. März 2019.

Statistisches Bundesamt (2019b). Anzahl der Suizide nach Altersgruppen. https://www. destatis.de/DE/Themen/Gesellschaft-Umwelt/Gesundheit/Todesursachen/Tabellen/sterbefaelle-suizid-erwachsene-kinder.html. Zugegriffen: 24. März 2019.

Statistisches Bundesamt (2019c). Zahl der Todesfälle im Jahr 2016 um 1,5 \% gesunken. https://www.destatis.de/DE/Themen/Gesellschaft-Umwelt/Gesundheit/Todesursachen/ todesfaelle-2016.html. Zugegriffen: 24. März 2019.

Straus, F., Höfer, R., Nuchholz, W., \& Gmür, W. (1987). Die Bewältigung familiärer Probleme im sozialen Netzwerk. Überlegungen zur Praxisrelevanz der Netzwerkperspektive in der Familienarbeit. In H. Keupp \& B. Röhrle (Hrsg.), Soziale Netzwerke (S. 178-198). New York: Campus.

Turner, R. J., \& Marino, F. (1994). Social support and social structure: A descriptive epidemiology. Journal of Health and Social Behavior, 35(3), 193-212.

Umberson, D., Wortman, C. B., \& Kessler, R. C. (1992). Widowhood and depression: Explaining long-term gender differences in vulnerability. Journal of Health and Social Behavior, 33(1), 10-24.

UNdata. (2017). Life expectancy. http://data.un.org/Search.aspx?q=life+expectancy. Zugegriffen: 27. März 2019.

Underwood, P. W. (2005). Sozialer Rückhalt: Versprechen und Wirklichkeit. In V. Hill Rice, (Hrsg.), Stress und Coping. Lehrbuch für Pflegepraxis und -wissenschaft (S. 419-444). Bern: Hans Huber.

Valente, T. (2012). Network interventions. Science, 337(6090), 49-53. 
Van Emmerik, I. H. (2006). Gender differences in the creation of different types of social capital: A multilevel study. Social Networks, 28(1), 24-37.

Veiel, H. O. F., \& Herrle, Johannes. (1991). Geschlechtsspezifische Strukturen sozialer Unterstützungsnetzwerke. Zeitschrift für Soziologie, 20(3), 237-245.

Vyncke, V., Hardyns, W., Peersman, W., Pauwels, L., Groenewegen, P., \& Willems, S. (2014). How equal is the relationship between individual social capital and psychological distress? A gendered analysis using cross-sectional data from Ghent (Belgium). BMC Public Health, 14, 960.

Walen, H. R., \& Lachman, M. E. (2000). Social support and strain from partner, family, and friends: Costs and benefits for men and women in adulthood. Journal of Social and Personal Relationships, 17(1), 5-30.

Wetterer, A. (2004). Konstruktion von Geschlecht: Reproduktionsweisen der Zweigeschlechtlichkeit. In R. Becker \& B. Kortendiek (Hrsg.), Handbuch Frauen- und Geschlechterforschung (S. 122-131). Wiesbaden: VS.

WHO. (2019). Probability of dying per 1000 live births Data by WHO region. http://apps. who.int/gho/data/view.main.CM1300R?lang=en. Zugegriffen: 25. Apr. 2019.

Winker, G., \& Degele, N. (2010). Intersektionalität. Zur Analyse sozialer Ungleichheiten (2. Aufl.). Bielefeld: Transcript.

Winter, S., Diamond, M., Green, J., Karasic, D., Reed, T., Whittle, S., \& Wylie, K. (2016). Transgender people: Health at the margins of society. The Lancet, 388(10042), 390-400.

Wirtschafts- und Sozialwissenschaftliches Institut (WSI) (2017). Verdienstabstand nach Erwerbsumfang und beruflicher Position 2017. https://www.boeckler.de/52852.htm. Zugegriffen: 28. März 2019.

Wirtschafts- und Sozialwissenschaftliches Institut (WSI) (2018). Frauen in Deutschland. https://www.boeckler.de/wsi_38957.htm. Zugegriffen: 28. März 2019.

Zeiher, J., Kuntz, B., \& Lange, C. (2017). Rauchen bei Erwachsenen in Deutschland. https://www.rki.de/DE/Content/Gesundheitsmonitoring/Gesundheitsberichterstattung/ GBEDownloadsJ/FactSheets/JoHM_2017_02_Rauchen_Erwachsene.pdf. Zugegriffen: 26. März 2019.

Open Access Dieses Kapitel wird unter der Creative Commons Namensnennung 4.0 International Lizenz (http://creativecommons.org/licenses/by/4.0/deed.de) veröffentlicht, welche die Nutzung, Vervielfältigung, Bearbeitung, Verbreitung und Wiedergabe in jeglichem Medium und Format erlaubt, sofern Sie den/die ursprünglichen Autor(en) und die Quelle ordnungsgemäß nennen, einen Link zur Creative Commons Lizenz beifügen und angeben, ob Änderungen vorgenommen wurden.

Die in diesem Kapitel enthaltenen Bilder und sonstiges Drittmaterial unterliegen ebenfalls der genannten Creative Commons Lizenz, sofern sich aus der Abbildungslegende nichts anderes ergibt. Sofern das betreffende Material nicht unter der genannten Creative Commons Lizenz steht und die betreffende Handlung nicht nach gesetzlichen Vorschriften erlaubt ist, ist für die oben aufgeführten Weiterverwendungen des Materials die Einwilligung des jeweiligen Rechteinhabers einzuholen.

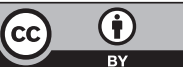

